\title{
Cosmology with a stiff matter era
}

\author{
Pierre-Henri Chavanis \\ Laboratoire de Physique Théorique, Université Paul Sabatier, 118 route de Narbonne 31062 Toulouse, France
}

\begin{abstract}
We provide a simple analytical solution of the Friedmann equations for a universe made of stiff matter, dust matter, and dark energy. A stiff matter era is present in the cosmological model of Zel'dovich (1972) where the primordial universe is assumed to be made of a cold gas of baryons. It also occurs in certain cosmological models where dark matter is made of relativistic self-gravitating Bose-Einstein condensates (BECs). When the energy density of the stiff matter is positive, the primordial universe is singular. It starts from a state with a vanishing scale factor and an infinite density. We consider the possibility that the energy density of the stiff matter is negative (anti-stiff matter). This happens, for example, when the BECs have an attractive self-interaction. In that case, the primordial universe is non-singular. It starts from a state in which the scale factor is finite and the energy density is equal to zero. For the sake of generality, we consider a cosmological constant of arbitrary sign. When the cosmological constant is positive, the universe asymptotically reaches a de Sitter phase where the scale factor increases exponentially rapidly. This can account for the accelerating expansion of the universe that we observe at present. When the cosmological constant is negative (anti-de Sitter), the evolution of the universe is cyclic. Therefore, depending on the sign of the energy density of the stiff matter and of the dark energy, we obtain singular and non-singular expanding or cyclic universes.
\end{abstract}

PACS numbers: 95.30.Sf, 95.35.+d, 98.80.-k

\section{INTRODUCTION}

In a seminal paper, Zel'dovich [1] introduced a cosmological model in which the very early universe is assumed to be made of a cold gas of baryons with a stiff equation of state $P=\epsilon$, where $P$ is the pressure and $\epsilon$ is the energy density. For a stiff matter fluid, the velocity of sound $c_{s}=\sqrt{P^{\prime}(\epsilon)} c$ is equal to the velocity of light [2]. In that case, the energy density decreases as $\epsilon \propto 1 / a^{6}$ where $a(t)$ is the scale factor. This phase, if it has ever existed, preceded the radiation era $\left(P=\epsilon / 3, \epsilon \propto a^{-4}\right)$, the dust matter era $\left(P=0, \epsilon \propto a^{-3}\right)$, and the dark energy era $\left(P=-\epsilon, \epsilon=\epsilon_{\Lambda}\right)$. A stiff matter era also occurs in certain cosmological models in which dark matter is made of relativistic self-gravitating BECs [3]. In that case, the energy density of the stiff matter can be positive or negative depending whether the self-interaction of the bosons is repulsive or attractive.

We show that when radiation is neglected, the Friedmann equations for a universe made of stiff matter, dust matter, and dark energy can be integrated analytically. This provides a simple cosmological model exhibiting a stiff matter era. We consider the general case where the energy density of the stiff fluid is positive or negative. The case of a stiff fluid with a positive energy density is very similar to the standard model of cosmology in the sense that the universe begins by a singularity at $t=0$ in which the scale factor is equal to zero while the energy density is infinite. Initially, the scale factor increases as $a(t) \propto t^{1 / 3}$ and the energy density decreases as $\epsilon(t) \propto t^{-2}$. Interestingly, the presence of a stiff fluid with a negative energy density (anti-stiff fluid) prevents the primordial singularity. In that case, we obtain a model of universe in which the initial scale factor is finite and the energy density is equal to zero. For the sake of gen- erality, we consider a positive or a negative cosmological constant. At late times, a dark fluid with a positive energy density leads to a de Sitter phase in which the scale factor increases exponentially rapidly. This can account for the present acceleration of the universe. By contrast, when the dark fluid has a negative energy density (antidark fluid) the evolution of the universe is cyclic.

The paper is organized as follows. In Sec. III, we consider a perfect fluid at $T=0$ described by a polytropic equation of state of the form $P=K \rho^{2}$ where $\rho$ is restmass density. We determine the relation between the energy density $\epsilon$ and the rest-mass density $\rho$ and obtain an explicit equation of state $P(\epsilon)$ relating the pressure to the energy density. At high energies, it reduces to a stiff equation of state $P \simeq \epsilon$ for which the velocity of sound is equal to the velocity of light. This equation of state describes a cold gas of baryons in Zel'dovich model [1] or a relativistic gas of BECs (in certain approximations) [3]. In Secs. III and IV we apply this equation of state to cosmology. This leads to a cosmological model exhibiting a primordial stiff matter era, followed by a radiation era, a dust matter era, and a dark energy era. In Secs. V] [IX], we provide simple analytical solutions of the Friedmann equations for a universe comprising a stiff fluid. We consider four cases: (i) a singular expanding universe with $K \geq 0$ and $\Lambda \geq 0$; (ii) a non-singular expanding universe with $K \leq 0$ and $\Lambda \geq 0$; (iii) a singular cyclic universe with $K \geq 0$ and $\Lambda \leq 0$; (iv) a non-singular cyclic universe with $K \leq 0$ and $\Lambda \leq 0$.

\section{A STIFF EQUATION OF STATE}

In this section, we consider physical systems described by a stiff equation of state. 


\section{A. General results for a fluid at $T=0$}

The local form of the first law of thermodynamics can be written as

$$
d\left(\frac{\epsilon}{\rho}\right)=-P d\left(\frac{1}{\rho}\right)+T d\left(\frac{s}{\rho}\right),
$$

where $\rho$ is the mass density and $s$ is the entropy density in the rest frame. For a system at $T=0$, the first law of thermodynamics reduces to

$$
d \epsilon=\frac{P+\epsilon}{\rho} d \rho .
$$

For a given equation of state, Eq. (2) can be integrated to obtain the relation between the energy density $\epsilon$ and the rest-mass density $\rho$. For example, let us assume that the equation of state is prescribed under the form $P=P(\rho)$. In that case, Eq. (2) reduces to the first order linear differential equation

$$
\frac{d \epsilon}{d \rho}-\frac{1}{\rho} \epsilon=\frac{P(\rho)}{\rho} .
$$

Using the method of the variation of the constant, we obtain

$$
\epsilon=A \rho c^{2}+\rho \int^{\rho} \frac{P\left(\rho^{\prime}\right)}{\rho^{\prime 2}} d \rho^{\prime}
$$

where $A$ is a constant of integration. For an equation of state $P(\rho)$ such that $P \sim \rho^{\gamma}$ with $\gamma>1$ when $\rho \rightarrow 0$, we determine the constant $A$ in Eq. (4) by requiring that $\epsilon \sim \rho c^{2}$ when $\rho \rightarrow 0$. This gives

$$
\epsilon=\rho c^{2}+\rho \int_{0}^{\rho} \frac{P\left(\rho^{\prime}\right)}{\rho^{\prime 2}} d \rho^{\prime}=\rho c^{2}+u(\rho) .
$$

We note that $u(\rho)$ may be interpreted as an internal energy [4].

\section{B. Polytrope $n=1$}

We consider the equation of state

$$
P=K \rho^{2}
$$

corresponding to a polytrope of index $n=1[\underline{5}]$. In that case, Eq. (5) reduces to

$$
\epsilon=\rho c^{2}+P=\rho c^{2}+K \rho^{2} .
$$

This equation can be reversed to give

$$
\rho=\frac{c^{2}}{2 K}\left(\sqrt{1+\frac{4 K \epsilon}{c^{4}}}-1\right) .
$$

Combining Eqs. (6) and (8), we obtain the relation between the pressure and the energy density

$$
P=\frac{c^{4}}{4 K}\left(\sqrt{1+\frac{4 K \epsilon}{c^{4}}}-1\right)^{2}
$$

For $\epsilon \rightarrow 0$ (non-relativistic limit), we get

$$
\epsilon \sim \rho c^{2}, \quad P \sim \frac{K}{c^{4}} \epsilon^{2}, \quad P=K \rho^{2} .
$$

This is a polytropic equation of state $P=K\left(\epsilon / c^{2}\right)^{2}$ of index $n=1$. For $\epsilon \rightarrow+\infty$ (ultra-relativistic limit), we get

$$
\epsilon \sim K \rho^{2}, \quad P \sim \epsilon, \quad P=K \rho^{2}
$$

This is a linear equation of state $P=\epsilon$ that is called "stiff" because the velocity of sound is equal to the velocity of light $\left(c_{s}=c\right)$.

For the equation of state (9), the velocity of sound is given by

$$
\frac{c_{s}^{2}}{c^{2}}=P^{\prime}(\epsilon)=1-\frac{1}{\sqrt{1+4 K \epsilon / c^{4}}} .
$$

We always have $c_{s}<c$. For $\epsilon \rightarrow+\infty, c_{s} \rightarrow c$.

\section{Gas of baryons interacting through a vector meson field}

Zel'dovich [1, 2] introduced a cosmological model in which the primordial universe is made of a gas of baryons interacting through a vector meson field and showed that the equation of state of this system is of the form of Eq. (6) with a polytropic constant

$$
K=\frac{g^{2} h^{2}}{2 \pi m_{m}^{2} m_{b}^{2} c^{2}},
$$

where $g$ is the baryon charge, $m_{m}$ is the meson mass, and $m_{b}$ is the baryon mass. Zel'dovich [2] introduced this equation of state as an example to show how the speed of sound could approach the speed of light at very high pressures and densities.

Zel'dovich [1, 2] also mentions that the complete equation of state of his model is of the form

$$
P=K \rho^{2}+K^{\prime} \rho^{4 / 3}
$$

where $K$ is given by Eq. (13) and the second term accounts for quantum (Fermi) corrections. For the equation of state (14), we find from Eq. (5) that the relation between the energy density and the rest-mass density is

$$
\epsilon=\rho c^{2}+K \rho^{2}+3 K^{\prime} \rho^{4 / 3}
$$




\section{Relativistic self-gravitating BECs}

Some authors have proposed that dark matter may be made of self-gravitating BECs with short-range interactions 6 22]. In the TF approximation, a BEC is equivalent to a fluid with an equation of state of the form of Eq. (6) with a polytropic constant

$$
K=\frac{2 \pi \hbar^{2} a_{s}}{m^{3}}
$$

where $m$ is the mass of the bosons and $a_{s}$ is their scattering length. This equation of state can be derived from the classical Gross-Pitaevskii equation [23, 24] after writing it under the form of fluid equation by using the Madelung transformation [25]. It is therefore a nonrelativistic equation of state that, in principle, is not valid in the relativistic regime. Nevertheless, we can consider a partially-relativistic model in which we use the classical equation of state (6) with the relativistic relation (7) between the energy density and the rest-mass density. This approximation has been considered in [3] .

A feature of BECs is that the scattering length $a_{s}$ can be positive or negative [26]. Positive values of $a_{s}$ correspond to repulsive interactions and negative values of $a_{s}$ correspond to attractive interactions. When $a_{s}$ is positive, the pressure is positive and when $a_{s}$ is negative the pressure is negative. We shall consider the two possibilities in the following.

\section{COSMOLOGY WITH A STIFF FLUID}

In this section, we derive the main equations governing the evolution of a universe described by a stiff equation of state of the form of Eq. (9).

\section{A. The Friedmann equations}

We assume that the universe is homogeneous and isotropic, and contains a uniform perfect fluid of energy density $\epsilon(t)$ and isotropic pressure $P(t)$. The radius of curvature of the 3-dimensional space, or scale factor, is noted $a(t)$ and the curvature of space is noted $k$. The universe is closed if $k>0$, flat if $k=0$, and open if $k<0$. We assume that the universe is flat $(k=0)$ in agreement with the observations of the cosmic microwave background (CMB) [27]. In that case, the Einstein equations can be written as [28]:

$$
\begin{gathered}
\frac{d \epsilon}{d t}+3 \frac{\dot{a}}{a}(\epsilon+P)=0 \\
\frac{\ddot{a}}{a}=-\frac{4 \pi G}{3 c^{2}}(\epsilon+3 P)+\frac{\Lambda}{3}, \\
H^{2}=\left(\frac{\dot{a}}{a}\right)^{2}=\frac{8 \pi G}{3 c^{2}} \epsilon+\frac{\Lambda}{3},
\end{gathered}
$$

where we have introduced the Hubble parameter $H=$ $\dot{a} / a$ and accounted for a possible non-zero cosmological constant $\Lambda$. The cosmological constant is equivalent to a dark energy fluid with a constant density

$$
\epsilon_{\Lambda}=\rho_{\Lambda} c^{2}=\frac{\Lambda c^{2}}{8 \pi G}
$$

and an equation of state $P=-\epsilon$. Eqs. (17)-(19) are the well-known Friedmann equations describing a nonstatic universe. Among these three equations, only two are independent. The first equation can be viewed as an equation of continuity. For a given barotropic equation of state $P=P(\epsilon)$, it determines the relation between the energy density $\epsilon$ and the scale factor $a$. Then, the evolution of the scale factor $a(t)$ is given by Eq. (19).

\section{B. General results for a fluid at $T=0$}

We assume that the universe is made of a fluid at $T=0$ with an equation of state $P(\rho)$. In that case, the relation between the energy density $\epsilon$ and the rest-mass density $\rho$ is given by the first law of relativistic thermodynamics, Eq. (2). Combining this relation with the continuity equation (17), we get

$$
\frac{d \rho}{d t}+3 \frac{\dot{a}}{a} \rho=0 .
$$

We note that this equation is exact for a fluid at $T=0$ and that it does not depend on the explicit form of the equation of state $P(\rho)$. It can be integrated into

$$
\rho=\rho_{0}\left(\frac{a_{0}}{a}\right)^{3},
$$

where $\rho_{0}$ is the present value of the rest-mass density and $a_{0}$ is the present value of the scale factor.

\section{Polytrope $n=1$}

We now consider the equation of state (6). As we have seen, this equation of state appears in the cosmological model of Zel'dovich [1] in which the early universe is made of a cold gas of baryons. This equation of state also appears in certain cosmological models in which dark matter is made of relativistic BECs [3]. For the equation of state (6), Eq. (5) can be integrated easily and the relation between the energy density and the rest-mass density is given by Eq. (77). Combining Eqs. (77) and (22), we get

$$
\epsilon=\rho_{0} c^{2}\left(\frac{a_{0}}{a}\right)^{3}+K \rho_{0}^{2}\left(\frac{a_{0}}{a}\right)^{6} .
$$

This relation can also be obtained by solving the continuity equation (17) with the equation of state (9), see Appendix D of [3]. 
In the early universe $(a \rightarrow 0)$, we have

$$
\epsilon \sim K \rho_{0}^{2}\left(\frac{a_{0}}{a}\right)^{6}, \quad \epsilon \sim K \rho^{2}, \quad P \sim \epsilon .
$$

These equations describe a stiff fluid $(P=\epsilon)$ for which the velocity of sound is equal to the velocity of light.

In the late universe $(a \rightarrow+\infty)$, we have

$$
\epsilon \sim \rho_{0} c^{2}\left(\frac{a_{0}}{a}\right)^{3}, \quad \epsilon \sim \rho c^{2}, \quad P \sim \frac{K}{c^{4}} \epsilon^{2} .
$$

These equations describe a fluid with a polytropic equation of state of index $n=1\left(P=K \epsilon^{2} / c^{4}\right)$. For very large values of the scale factor, we recover the results of the CDM model $(P=0)$ since $\epsilon \propto a^{-3}$.

When combined with the Friedmann equation (19), Eq. (23) describes a model of universe exhibiting a stiff matter phase $\left(\epsilon \propto a^{-6}\right)$, a dust matter phase $\left(\epsilon \propto a^{-3}\right)$, and a dark energy phase $\left(\epsilon \sim \rho_{\Lambda} c^{2}\right)$.

\section{A more general equation of state}

If we consider the more general equation of state (14) suggested by Zel'dovich [1, 2], the relation between the energy density and the rest-mass density is given by Eq. (15). Using Eq. (22), we get

$$
\epsilon=\rho_{0} c^{2}\left(\frac{a_{0}}{a}\right)^{3}+K \rho_{0}^{2}\left(\frac{a_{0}}{a}\right)^{6}+3 K^{\prime} \rho_{0}^{4 / 3}\left(\frac{a_{0}}{a}\right)^{4} .
$$

When combined with the Friedmann equation (19), Eq. (26) describes a model of universe exhibiting a stiff matter phase $\left(\epsilon \propto a^{-6}\right)$, a radiation phase $\left(\epsilon \propto a^{-4}\right)$, a dust matter phase $\left(\epsilon \propto a^{-3}\right)$, and a dark energy phase $\left(\epsilon \sim \rho_{\Lambda} c^{2}\right)$.

\section{THE FRIEDMANN EQUATIONS FOR A UNIVERSE PRESENTING A STIFF MATTER ERA}

In this section, we assume that the universe is made of one or several fluids each of them described by a linear equation of state $P=\alpha \epsilon$. The equation of continuity (17) implies that the energy density is related to the scale factor by $\epsilon=\epsilon_{0}\left(a_{0} / a\right)^{3(1+\alpha)}$, where the subscript 0 denotes present-day values of the quantities. A linear equation of state can describe dust matter $\left(\alpha=0, \epsilon_{m} \propto a^{-3}\right)$, radiation $\left(\alpha=1 / 3, \epsilon_{\text {rad }} \propto a^{-4}\right)$, stiff matter $(\alpha=1$, $\left.\epsilon_{s} \propto a^{-6}\right)$, vacuum energy $\left(\alpha=-1, \epsilon=\epsilon_{P}\right)$, and dark energy $\left(\alpha=-1, \epsilon=\epsilon_{\Lambda}\right)$.

More specifically, we consider a universe made of stiff matter, radiation, dust matter and dark energy treated as non-interacting species. Summing the contribution of each species, the total energy density can be written as

$$
\epsilon=\frac{\epsilon_{s, 0}}{\left(a / a_{0}\right)^{6}}+\frac{\epsilon_{r a d, 0}}{\left(a / a_{0}\right)^{4}}+\frac{\epsilon_{m, 0}}{\left(a / a_{0}\right)^{3}}+\epsilon_{\Lambda} .
$$

In this model, the stiff matter dominates in the early universe. This is followed by the radiation era, by the dust matter era and, finally, by the dark energy era. Writing $\epsilon_{\alpha, 0}=\Omega_{\alpha, 0} \epsilon_{0}$ for each species, we get

$$
\frac{\epsilon}{\epsilon_{0}}=\frac{\Omega_{s, 0}}{\left(a / a_{0}\right)^{6}}+\frac{\Omega_{r a d, 0}}{\left(a / a_{0}\right)^{4}}+\frac{\Omega_{m, 0}}{\left(a / a_{0}\right)^{3}}+\Omega_{\Lambda, 0} .
$$

The last term in Eq. (28), corresponding to the dark energy, is equivalent to the cosmological constant in Eq. (19). For the sake of generality, we consider the case of a positive $\left(\Omega_{\Lambda, 0} \geq 0\right)$ or negative $\left(\Omega_{\Lambda, 0} \leq 0\right)$ cosmological constant $\Lambda$. When the radiation term is neglected, Eq. (28) is equivalent to Eq. (23) obtained from the equation of state (6) and (13) proposed by Zel'dovich [1, 2] or from the equation of state (6) and (16) corresponding to a relativistic BEC [3]. On the other hand, Eq. (28) with the radiation term included is equivalent to Eq. (26) obtained from the more general equation of state (14) and (13) suggested by Zel'dovich [1, 2]. Comparing Eqs. (23) and (28), we see that a positive value of $K$ corresponds to a positive energy density of the stiff matter while a negative value of $K$ corresponds to a negative energy density of the stiff matter. We shall therefore consider the two possibilities $\Omega_{s, 0} \geq 0$ and $\Omega_{s, 0} \leq 0$. However, we take $\Omega_{m, 0} \geq 0$ and $\Omega_{\text {rad, } 0} \geq 0$.

Using Eq. (28), the Friedmann equation (19) takes the form

$$
\frac{H}{H_{0}}=\sqrt{\frac{\Omega_{s, 0}}{\left(a / a_{0}\right)^{6}}+\frac{\Omega_{\text {rad,0}}}{\left(a / a_{0}\right)^{4}}+\frac{\Omega_{m, 0}}{\left(a / a_{0}\right)^{3}}+\Omega_{\Lambda, 0}}
$$

with $\Omega_{s, 0}+\Omega_{\text {rad }, 0}+\Omega_{m, 0}+\Omega_{\Lambda, 0}=1$ and $H_{0}=$ $\left(8 \pi G \epsilon_{0} / 3 c^{2}\right)^{1 / 2}$. We note the relation

$$
\frac{\epsilon}{\epsilon_{0}}=\left(\frac{H}{H_{0}}\right)^{2}
$$

The evolution of the scale factor is given by

$$
\int_{a_{i} / a_{0}}^{a / a_{0}} \frac{d x}{x \sqrt{\frac{\Omega_{s, 0}}{x^{6}}+\frac{\Omega_{\text {rad,0 }}}{x^{4}}+\frac{\Omega_{m, 0}}{x^{3}}+\Omega_{\Lambda, 0}}}=H_{0} t
$$

where $a_{i}$ is the initial value of the scale factor determined below.

In Secs. VIVIII, we ignore radiation $\left(\Omega_{\text {rad }, 0}=0\right)$ and consider a universe made of stiff matter, dust matter, and dark energy. In that case, the Friedmann equation (31) reduces to

$$
\int_{a_{i} / a_{0}}^{a / a_{0}} \frac{d x}{x \sqrt{\frac{\Omega_{s, 0}}{x^{6}}+\frac{\Omega_{m, 0}}{x^{3}}+\Omega_{\Lambda, 0}}}=H_{0} t
$$

which can be integrated analytically. In Secs. IX, we provide some particular analytical solutions of Eq. (31) in the case where the radiation is taken into account. 


\section{THE CASE $\Omega_{s, 0} \geq 0$ AND $\Omega_{\Lambda, 0} \geq 0$}

We first consider the case of a positive stiff energy density $\left(\Omega_{s, 0} \geq 0\right)$ and a positive cosmological constant $\left(\Omega_{\Lambda, 0} \geq 0\right)$. The total energy density is

$$
\frac{\epsilon}{\epsilon_{0}}=\frac{\Omega_{s, 0}}{\left(a / a_{0}\right)^{6}}+\frac{\Omega_{m, 0}}{\left(a / a_{0}\right)^{3}}+\Omega_{\Lambda, 0} .
$$

The energy density starts from $\epsilon=+\infty$ at $a=a_{i}=0$, decreases, and tends to $\epsilon_{\Lambda}$ for $a \rightarrow+\infty$. The relation between the energy density and the scale factor is shown in Fig. 1. The proportions of stiff matter, dust matter and dark energy as a function of the scale factor are shown in Fig. 2.

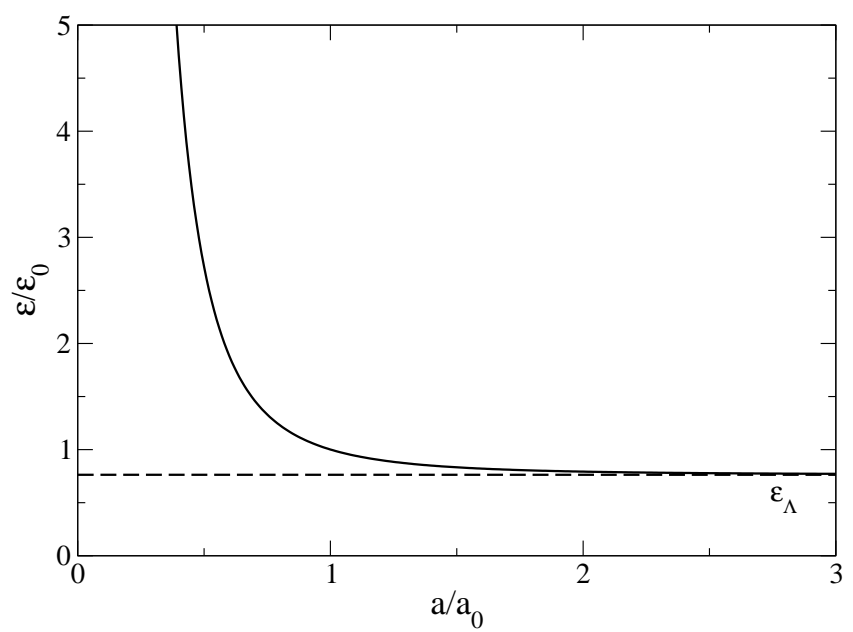

FIG. 1: Energy density as a function of the scale factor. We have taken $\Omega_{m, 0}=0.237, \Omega_{\Lambda, 0}=0.763$, and $\Omega_{s, 0}=10^{-3}$ (here and in the following figures, we have chosen a relatively large value of the density of stiff matter $\Omega_{s, 0}$ for a better illustration of the results).

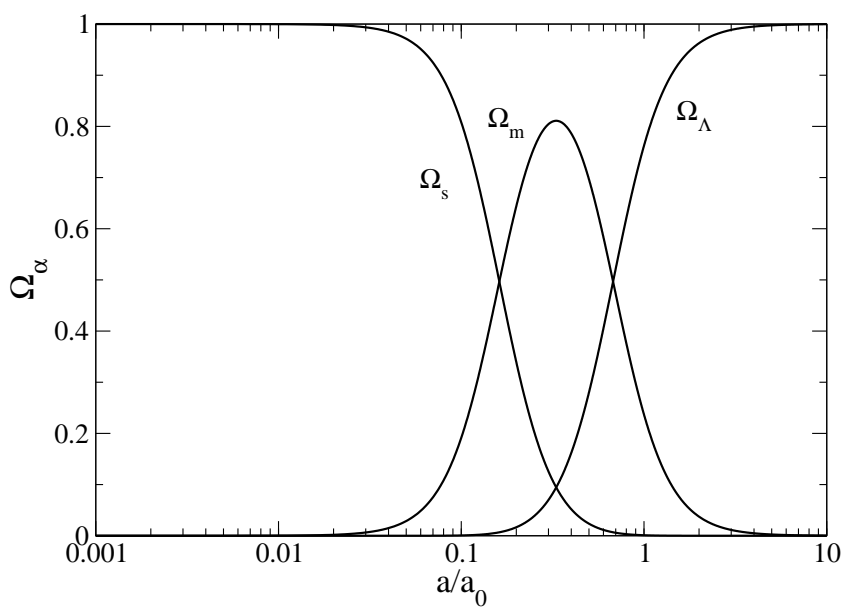

FIG. 2: Evolution of the proportion $\Omega_{\alpha}=\epsilon_{\alpha} / \epsilon$ of the different components of the universe with the scale factor.

\section{A. Stiff matter, dust matter, and dark energy}

We consider a universe made of stiff matter, dust matter, and dark energy. Using the identity

$$
\begin{gathered}
\int \frac{d x}{x \sqrt{\frac{a}{x^{3}}+\frac{b}{x^{6}}+c}} \\
=\frac{1}{3 \sqrt{c}} \ln \left[a+2 c x^{3}+2 \sqrt{c} \sqrt{b+a x^{3}+c x^{6}}\right],
\end{gathered}
$$

Eq. (32) can be solved analytically to give

$$
\begin{aligned}
\frac{a}{a_{0}}=\left[\left(\frac{\Omega_{m, 0}}{\Omega_{\Lambda, 0}}\right.\right. & \left.+2 \sqrt{\frac{\Omega_{s, 0}}{\Omega_{\Lambda, 0}}}\right) \sinh ^{2}\left(\frac{3}{2} \sqrt{\Omega_{\Lambda, 0}} H_{0} t\right) \\
& \left.+\sqrt{\frac{\Omega_{s, 0}}{\Omega_{\Lambda, 0}}}\left(1-e^{-3 \sqrt{\Omega_{\Lambda, 0}}} H_{0} t\right)\right]^{1 / 3} .
\end{aligned}
$$

From Eq. (35), we can compute $H=\dot{a} / a$ leading to

$$
\begin{aligned}
\left(\frac{a}{a_{0}}\right)^{3} \frac{H}{H_{0}} & =\left(\frac{\Omega_{m, 0}}{2 \sqrt{\Omega_{\Lambda, 0}}}+\sqrt{\Omega_{s, 0}}\right) \sinh \left(3 \sqrt{\Omega_{\Lambda, 0}} H_{0} t\right) \\
& +\sqrt{\Omega_{s, 0}} e^{-3 \sqrt{\Omega_{\Lambda, 0}}} H_{0} t
\end{aligned}
$$

The energy density $\epsilon / \epsilon_{0}$ is given by Eq. (30) where $H / H_{0}$ can be obtained from Eq. (36) with Eq. (35).

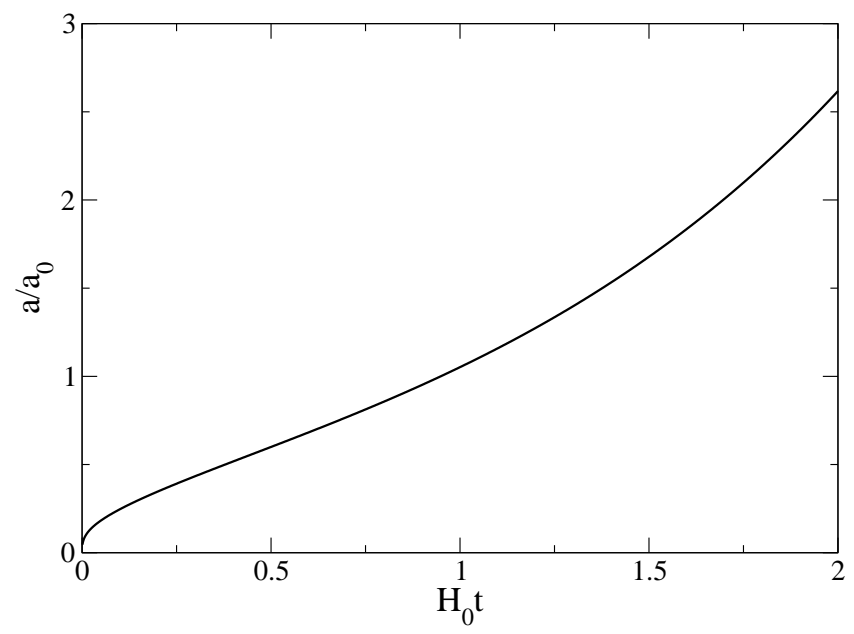

FIG. 3: Evolution of the scale factor as a function of time.

At $t=0$ the universe starts from a singular state at which the scale factor $a=0$ while the energy density $\epsilon=$ $+\infty$. The scale factor increases with time. For $t \rightarrow+\infty$, we obtain

$$
\frac{a}{a_{0}} \sim\left(\frac{\Omega_{m, 0}}{\Omega_{\Lambda, 0}}+2 \sqrt{\frac{\Omega_{s, 0}}{\Omega_{\Lambda, 0}}}\right)^{1 / 3} \frac{1}{2^{2 / 3}} e^{\sqrt{\Omega_{\Lambda, 0}}} H_{0} t .
$$

The energy density decreases with time and tends to $\epsilon_{\Lambda}$ for $t \rightarrow+\infty$. The expansion is decelerating during the 


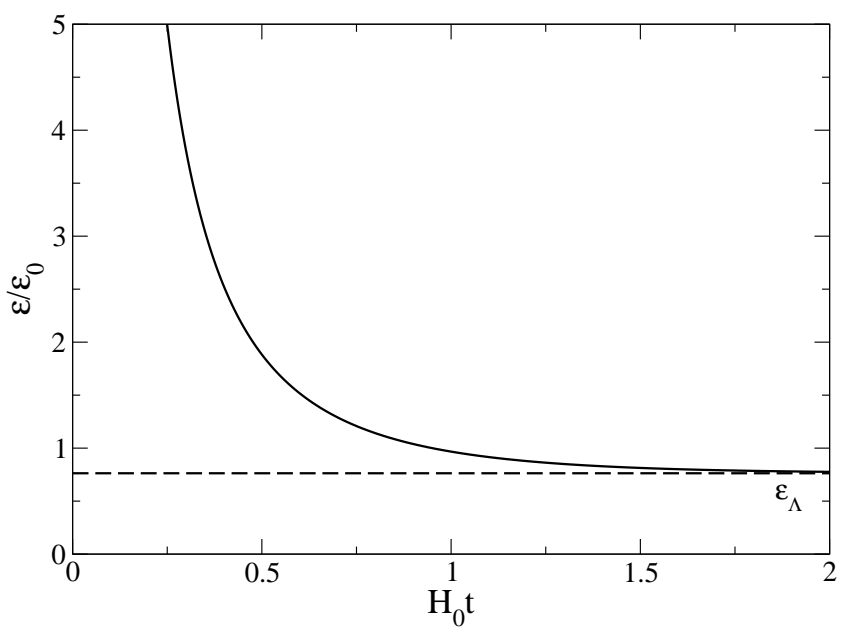

FIG. 4: Evolution of the energy density as a function of time.

stiff matter era and the dust matter era while it is accelerating during the dark energy era. The temporal evolutions of the scale factor and of the energy density are shown in Figs. 3 and 4.

\section{B. Stiff matter and dust matter}

We consider a universe made of stiff matter and dust matter. In the absence of dark energy $\left(\Omega_{\Lambda, 0}=0\right)$, using the identity

$$
\int \frac{d x}{x \sqrt{\frac{a}{x^{3}}+\frac{b}{x^{6}}}}=\frac{2}{3 a} \sqrt{b+a x^{3}}
$$

we obtain

$$
\begin{gathered}
\frac{a}{a_{0}}=\left(\frac{9}{4} \Omega_{m, 0} H_{0}^{2} t^{2}+3 \sqrt{\Omega_{s, 0}} H_{0} t\right)^{1 / 3}, \\
\frac{\epsilon}{\epsilon_{0}}=\frac{4}{9 H_{0}^{2} t^{2}}\left(\frac{1+\frac{2 \sqrt{\Omega_{s, 0}}}{3 \Omega_{m, 0} H_{0} t}}{1+\frac{4 \sqrt{\Omega_{s, 0}}}{3 \Omega_{m, 0} H_{0} t}}\right)^{2} .
\end{gathered}
$$

\section{Stiff matter and dark energy}

We consider a universe made of stiff matter and dark energy. In the absence of matter $\left(\Omega_{m, 0}=0\right)$, using the identity

$$
\int \frac{d x}{x \sqrt{\frac{b}{x^{6}}+c}}=\frac{1}{3 \sqrt{c}} \ln \left[2 c x^{3}+2 \sqrt{c} \sqrt{b+c x^{6}}\right]
$$

or setting $X=b / c x^{6}$ and using the identity

$$
\int \frac{d X}{X \sqrt{X+1}}=\ln \left(\frac{\sqrt{1+X}-1}{\sqrt{1+X}+1}\right)
$$

we get

$$
\begin{gathered}
\frac{a}{a_{0}}=\left(\frac{\Omega_{s, 0}}{\Omega_{\Lambda, 0}}\right)^{1 / 6} \sinh ^{1 / 3}\left(3 \sqrt{\Omega_{\Lambda, 0}} H_{0} t\right) \\
\frac{\epsilon}{\epsilon_{0}}=\frac{\Omega_{\Lambda, 0}}{\tanh ^{2}\left(3 \sqrt{\Omega_{\Lambda, 0}} H_{0} t\right)} .
\end{gathered}
$$

\section{Stiff matter}

We consider a universe made of stiff matter. In the absence of dust matter and dark energy $\left(\Omega_{m, 0}=\Omega_{\Lambda, 0}=\right.$ $0)$, we find that

$$
\frac{a}{a_{0}}=\left(3 \sqrt{\Omega_{s, 0}} H_{0} t\right)^{1 / 3}, \quad \frac{\epsilon}{\epsilon_{0}}=\frac{1}{9 H_{0}^{2} t^{2}} .
$$

\section{E. Dust matter and dark energy}

We consider a universe made of dust matter and dark energy. In the absence of stiff matter $\left(\Omega_{s, 0}=0\right)$, using the identity

$$
\int \frac{d x}{x \sqrt{\frac{a}{x^{3}}+c}}=\frac{1}{3 \sqrt{c}} \ln \left[a+2 c x^{3}+2 \sqrt{c} \sqrt{a x^{3}+c x^{6}}\right],
$$

or setting $X=a / c x^{3}$ and using identity (42), we obtain

$$
\begin{gathered}
\frac{a}{a_{0}}=\left(\frac{\Omega_{m, 0}}{\Omega_{\Lambda, 0}}\right)^{1 / 3} \sinh ^{2 / 3}\left(\frac{3}{2} \sqrt{\Omega_{\Lambda, 0}} H_{0} t\right), \\
\frac{\epsilon}{\epsilon_{0}}=\frac{\Omega_{\Lambda, 0}}{\tanh ^{2}\left(\frac{3}{2} \sqrt{\Omega_{\Lambda, 0}} H_{0} t\right)} .
\end{gathered}
$$

This corresponds to the $\Lambda$ CDM model.

\section{F. Dark energy}

We consider a universe made of dark energy. In the absence of stiff matter and dust matter $\left(\Omega_{s, 0}=\Omega_{m, 0}=\right.$ $0)$, we obtain

$$
a(t)=a(0) e^{\sqrt{\frac{\Lambda}{3}} t}, \quad \epsilon=\epsilon_{\Lambda}
$$

This is de Sitter's solution.

\section{G. Dust matter}

We consider a universe made of dust matter. In the absence of stiff matter and dark energy $\left(\Omega_{s, 0}=\Omega_{\Lambda, 0}=\right.$ $0)$, we obtain

$$
\frac{a}{a_{0}}=\left(\frac{9}{4} \Omega_{m, 0} H_{0}^{2} t^{2}\right)^{1 / 3}, \quad \frac{\epsilon}{\epsilon_{0}}=\frac{4}{9 H_{0}^{2} t^{2}} .
$$

This is the Einstein-de Sitter (EdS) solution. 


\section{THE CASE $\Omega_{s, 0} \leq 0$ AND $\Omega_{\Lambda, 0} \geq 0$}

We consider the case of a negative stiff energy density $\left(\Omega_{s, 0} \leq 0\right)$ and a positive cosmological constant $\left(\Omega_{\Lambda, 0} \geq\right.$ $0)$. The total energy density is

$$
\frac{\epsilon}{\epsilon_{0}}=-\frac{\left|\Omega_{s, 0}\right|}{\left(a / a_{0}\right)^{6}}+\frac{\Omega_{m, 0}}{\left(a / a_{0}\right)^{3}}+\Omega_{\Lambda, 0} .
$$

The energy density is positive for $a \geq a_{i}$ with

$$
\frac{a_{i}}{a_{0}}=\left(\frac{-\Omega_{m, 0}+\sqrt{\Delta}}{2 \Omega_{\Lambda, 0}}\right)^{1 / 3}
$$

where we have defined

$$
\Delta=\Omega_{m, 0}^{2}+4 \Omega_{\Lambda, 0}\left|\Omega_{s, 0}\right| .
$$

The energy density starts from $\epsilon=0$ at $a=a_{i}$, increases, reaches a maximum at

$$
\frac{a_{*}}{a_{0}}=\left(\frac{2\left|\Omega_{s, 0}\right|}{\Omega_{m, 0}}\right)^{1 / 3}, \quad \frac{\epsilon_{*}}{\epsilon_{0}}=\frac{\Delta}{4\left|\Omega_{s, 0}\right|},
$$

decreases, and tends to $\epsilon_{\Lambda}$ for $a \rightarrow+\infty$. The relation between the energy density and the scale factor is shown in Fig. 5. The proportions of stiff matter, dust matter and dark energy as a function of the scale factor are shown in Fig. 6.

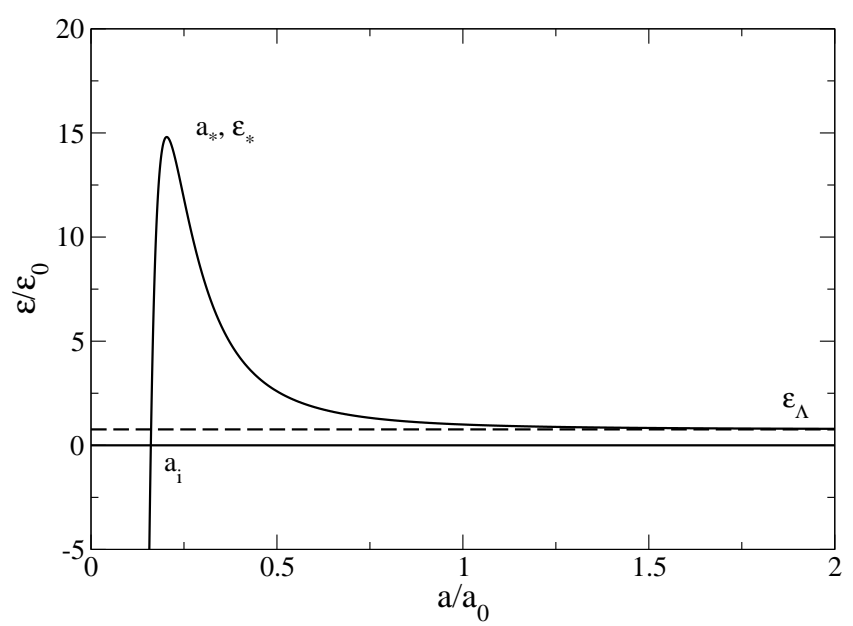

FIG. 5: Energy density as a function of the scale factor. We have taken $\Omega_{m, 0}=0.237, \Omega_{\Lambda, 0}=0.763$, and $\Omega_{s, 0}=-10^{-3}$.

\section{A. Anti-stiff matter, dust matter, and dark energy}

We consider a universe made of anti-stiff matter, dust matter, and dark energy. Using the identity (34), Eq. (32) with $a_{i}$ given by Eq. (52) can be solved analytically to give

$$
\frac{a}{a_{0}}=\left[\frac{\sqrt{\Delta}}{2 \Omega_{\Lambda, 0}} \cosh \left(3 \sqrt{\Omega_{\Lambda, 0}} H_{0} t\right)-\frac{\Omega_{m, 0}}{2 \Omega_{\Lambda, 0}}\right]^{1 / 3} .
$$

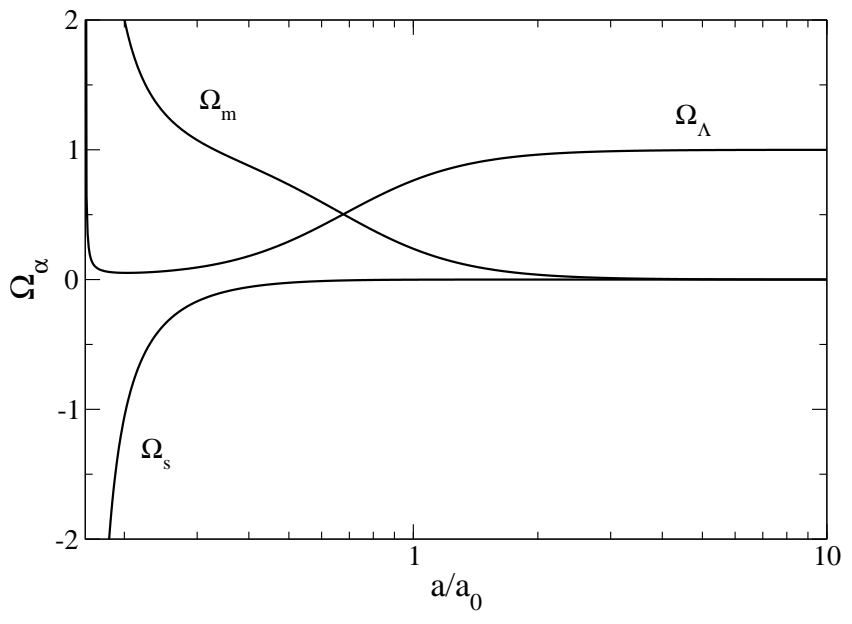

FIG. 6: Evolution of the proportion $\Omega_{\alpha}=\epsilon_{\alpha} / \epsilon$ of the different components of the universe with the scale factor.

From Eq. (55), we can compute $H=\dot{a} / a$ leading to

$$
\left(\frac{a}{a_{0}}\right)^{3} \frac{H}{H_{0}}=\frac{\sqrt{\Delta}}{2 \sqrt{\Omega_{\Lambda, 0}}} \sinh \left(3 \sqrt{\Omega_{\Lambda, 0}} H_{0} t\right) .
$$

The energy density $\epsilon / \epsilon_{0}$ is given by Eq. (30) where $H / H_{0}$ can be obtained from Eq. (56) with Eq. (55).

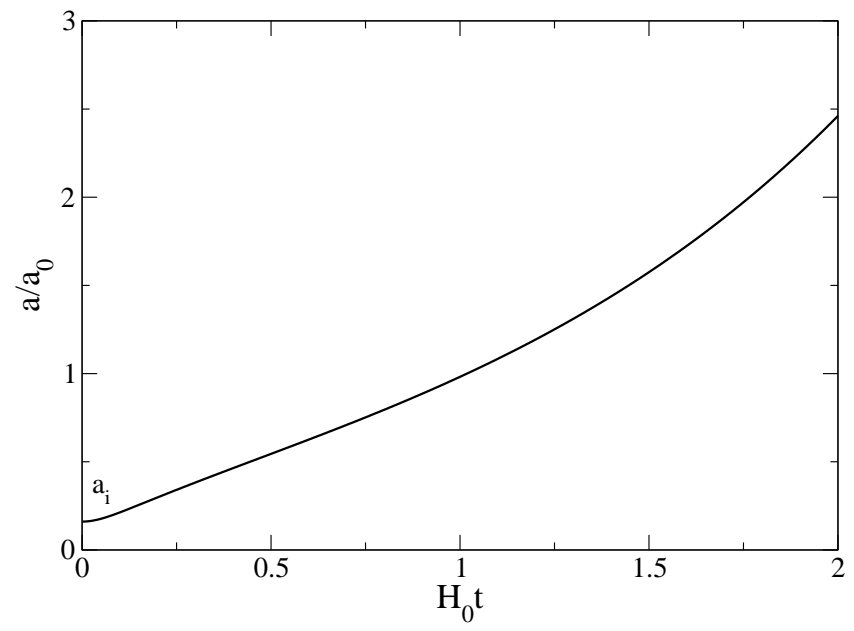

FIG. 7: Evolution of the scale factor as a function of time.

At $t=0$ the universe starts from a non-singular state at which the scale factor $a=a_{i}$ and the energy density $\epsilon=0$. The scale factor increases with time. For $t \rightarrow+\infty$, we obtain

$$
\frac{a}{a_{0}} \sim\left[\left(\frac{\Omega_{m, 0}}{\Omega_{\Lambda, 0}}\right)^{2}+4 \frac{\left|\Omega_{s, 0}\right|}{\Omega_{\Lambda, 0}}\right]^{1 / 6} \frac{1}{2^{2 / 3}} e^{\sqrt{\Omega_{\Lambda, 0}} H_{0} t} .
$$

The energy density increases, reaches its maximum value $\epsilon_{*}$ at $t=t_{*}$ where

$$
t_{*}=\frac{1}{3 \sqrt{\Omega_{\Lambda, 0}} H_{0}} \ln \left(\frac{\sqrt{\Delta}+\sqrt{4 \Omega_{\Lambda, 0}\left|\Omega_{s, 0}\right|}}{\Omega_{m, 0}}\right),
$$




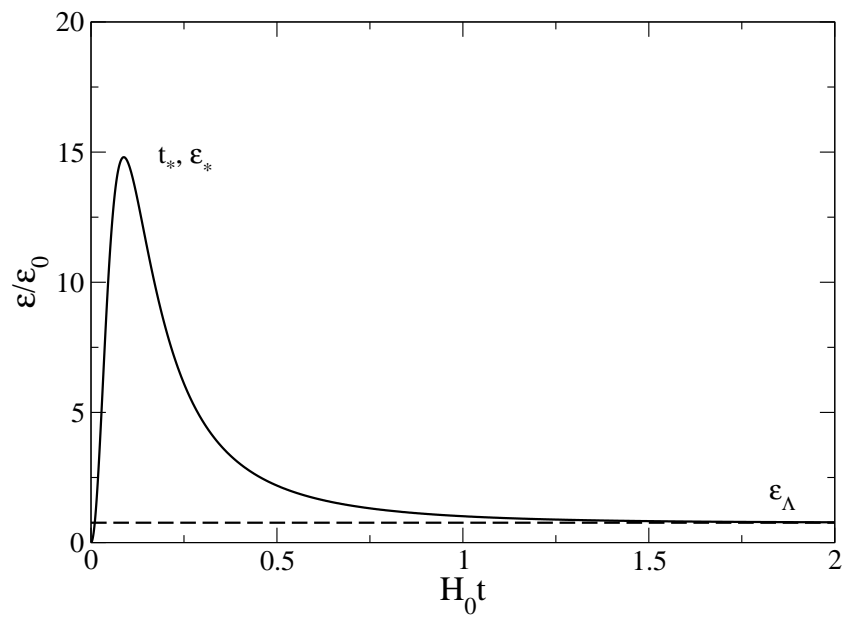

FIG. 8: Evolution of the energy density as a function of time.

decreases and tends to $\epsilon_{\Lambda}$ for $t \rightarrow+\infty$. The universe is accelerating during the anti-stiff matter era, decelerating during the dust matter era, and accelerating during the dark matter era. The temporal evolutions of the scale factor and of the energy density are shown in Figs. 7 and 8 .

\section{B. Anti-stiff matter and dust matter}

We consider a universe made of anti-stiff matter and dust matter. In the absence of dark energy $\left(\Omega_{\Lambda, 0}=0\right)$, using the identity (38), we obtain

$$
\begin{aligned}
\frac{a}{a_{0}} & =\left(\frac{9}{4} \Omega_{m, 0} H_{0}^{2} t^{2}+\frac{\left|\Omega_{s, 0}\right|}{\Omega_{m, 0}}\right)^{1 / 3}, \\
\frac{\epsilon}{\epsilon_{0}} & =\frac{4}{9 H_{0}^{2} t^{2}} \frac{1}{\left(1+\frac{4\left|\Omega_{s, 0}\right|}{9 \Omega_{m, 0}^{2} H_{0}^{2} t^{2}}\right)^{2}} .
\end{aligned}
$$

At $t=0$ the universe starts from a non-singular state at which the scale factor $a=a_{i}$ with

$$
\frac{a_{i}}{a_{0}}=\left(\frac{\left|\Omega_{s, 0}\right|}{\Omega_{m, 0}}\right)^{1 / 3}
$$

and the energy density $\epsilon=0$. The scale factor increases with time. The energy density increases, reaches its maximum value

$$
\frac{a_{*}}{a_{0}}=\left(\frac{2\left|\Omega_{s, 0}\right|}{\Omega_{m, 0}}\right)^{1 / 3}, \quad \frac{\epsilon_{*}}{\epsilon_{0}}=\frac{\Omega_{m, 0}^{2}}{4\left|\Omega_{s, 0}\right|},
$$

at

$$
t_{*}=\frac{2 \sqrt{\left|\Omega_{s, 0}\right|}}{3 \Omega_{m, 0} H_{0}},
$$

and decreases.

\section{Anti-stiff matter and dark energy}

We consider a universe made of anti-stiff matter and dark energy. In the absence of matter $\left(\Omega_{m, 0}=0\right)$, using the identities (41) and (42), we obtain

$$
\begin{gathered}
\frac{a}{a_{0}}=\left(\frac{\left|\Omega_{s, 0}\right|}{\Omega_{\Lambda, 0}}\right)^{1 / 6} \cosh ^{1 / 3}\left(3 \sqrt{\Omega_{\Lambda, 0}} H_{0} t\right), \\
\frac{\epsilon}{\epsilon_{0}}=\Omega_{\Lambda, 0} \tanh ^{2}\left(3 \sqrt{\Omega_{\Lambda, 0}} H_{0} t\right) .
\end{gathered}
$$

At $t=0$ the universe starts from a non-singular state at which the scale factor $a=a_{i}$ with

$$
\frac{a_{i}}{a_{0}}=\left(\frac{\left|\Omega_{s, 0}\right|}{\Omega_{\Lambda, 0}}\right)^{1 / 6},
$$

and the energy density $\epsilon=0$. The scale factor increases with time. The energy density increases with time and tends to $\epsilon_{\Lambda}$ for $t \rightarrow+\infty$.

\section{THE CASE $\Omega_{s, 0} \geq 0$ AND $\Omega_{\Lambda, 0} \leq 0$}

We consider the case of a positive stiff energy density $\left(\Omega_{s, 0} \geq 0\right)$ and a negative cosmological constant $\left(\Omega_{\Lambda, 0} \leq\right.$ $0)$. The total energy density is

$$
\frac{\epsilon}{\epsilon_{0}}=\frac{\Omega_{s, 0}}{\left(a / a_{0}\right)^{6}}+\frac{\Omega_{m, 0}}{\left(a / a_{0}\right)^{3}}-\left|\Omega_{\Lambda, 0}\right| .
$$

The energy density is positive for $a \leq a_{f}$ with

$$
\frac{a_{f}}{a_{0}}=\left(\frac{\Omega_{m, 0}+\sqrt{\Delta}}{2\left|\Omega_{\Lambda, 0}\right|}\right)^{1 / 3},
$$

where we have defined

$$
\Delta=\Omega_{m, 0}^{2}+4\left|\Omega_{\Lambda, 0}\right| \Omega_{s, 0} .
$$

The energy density starts from $\epsilon=+\infty$ at $a=0$, decreases, and reaches $\epsilon=0$ at $a=a_{f}$. The relation between the energy density and the scale factor is shown in Fig. 9. The proportions of stiff matter, dust matter and dark energy as a function of the scale factor are shown in Fig. 10.

\section{A. Stiff matter, dust matter, and anti-dark energy}

We consider a universe made of stiff matter, dust matter, and anti-dark energy. From Eqs. (35) and (36) with $\Omega_{\Lambda, 0}<0$ we get

$$
\begin{aligned}
& \frac{a}{a_{0}}=\left[\frac{\Omega_{m, 0}}{\left|\Omega_{\Lambda, 0}\right|} \sin ^{2}\left(\frac{3}{2} \sqrt{\left|\Omega_{\Lambda, 0}\right|} H_{0} t\right)\right. \\
& \left.+\sqrt{\frac{\Omega_{s, 0}}{\left|\Omega_{\Lambda, 0}\right|}} \sin \left(3 \sqrt{\left|\Omega_{\Lambda, 0}\right|} H_{0} t\right)\right]^{1 / 3},
\end{aligned}
$$




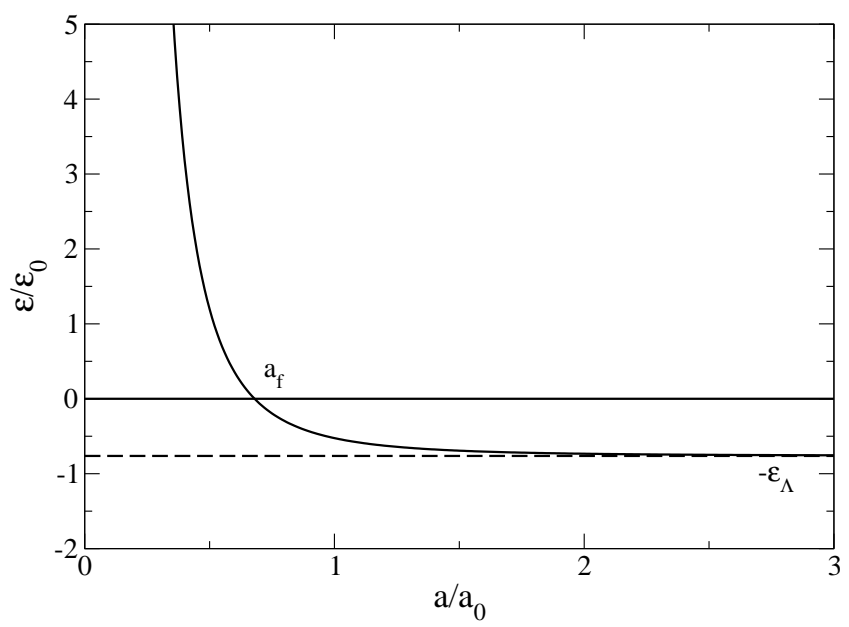

FIG. 9: Energy density as a function of the scale factor. We have taken $\Omega_{m, 0}=0.237, \Omega_{\Lambda, 0}=-0.763$, and $\Omega_{s, 0}=10^{-3}$.

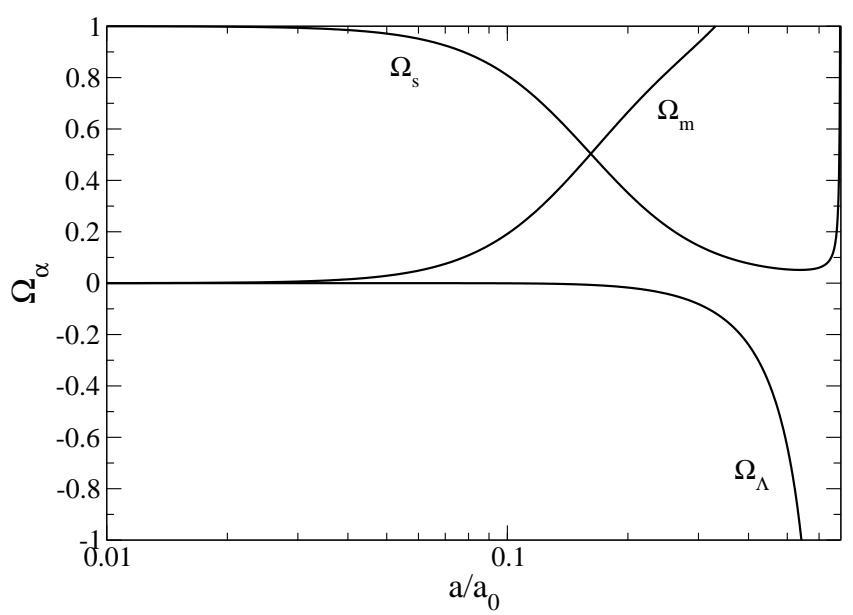

FIG. 10: Evolution of the proportion $\Omega_{\alpha}=\epsilon_{\alpha} / \epsilon$ of the different components of the universe with the scale factor.

and

$$
\begin{aligned}
\left(\frac{a}{a_{0}}\right)^{3} \frac{H}{H_{0}}= & \frac{\Omega_{m, 0}}{2 \sqrt{\left|\Omega_{\Lambda, 0}\right|}} \sin \left(3 \sqrt{\left|\Omega_{\Lambda, 0}\right|} H_{0} t\right) \\
& +\sqrt{\Omega_{s, 0}} \cos \left(3 \sqrt{\left|\Omega_{\Lambda, 0}\right|} H_{0} t\right) .
\end{aligned}
$$

The energy density is given by Eq. (30) where $H / H_{0}$ can be obtained from Eq. (71) with Eq. (70).

At $t=0$ the universe starts from a singularity at which the scale factor $a=0$ and the energy density $\epsilon=+\infty$. Between $t=0$ and $t=t_{1}$ where

$$
t_{1}=\frac{\pi-\tan ^{-1}\left(2 \sqrt{\Omega_{s, 0} \mid \Omega_{\Lambda, 0}} \mid / \Omega_{m, 0}\right)}{3 \sqrt{\left|\Omega_{\Lambda, 0}\right|} H_{0}},
$$

the energy density decreases from $\epsilon=+\infty$ to $\epsilon=0$ and the scale factor increases from $a=0$ to $a=a_{f}$. Between $t=t_{1}$ and $t=t_{2}=2 t_{1}$ the energy density increases from

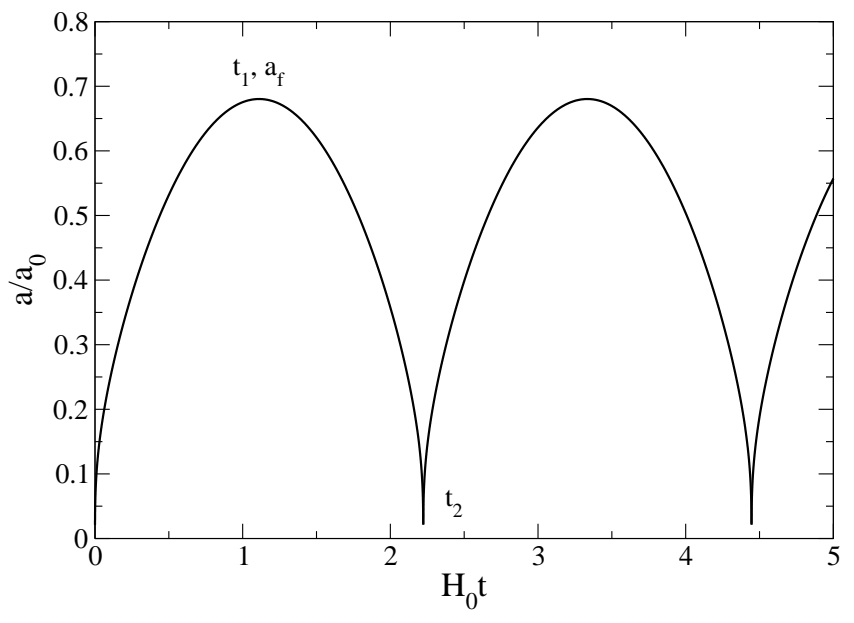

FIG. 11: Evolution of the scale factor as a function of time.

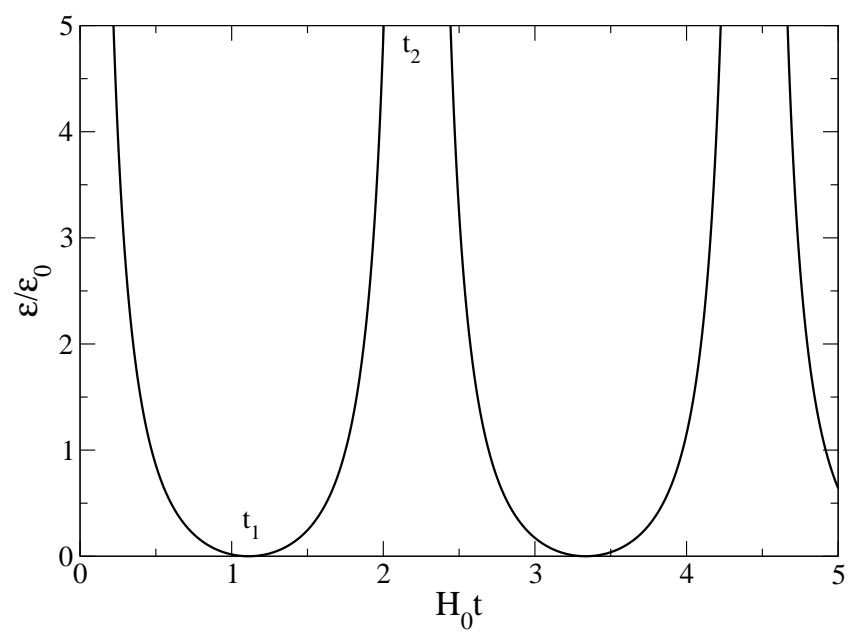

FIG. 12: Evolution of the energy density as a function of time.

$\epsilon=0$ to $\epsilon=+\infty$ and the scale factor decreases from $a=a_{f}$ to $a=0$. This process continues periodically with a period $t_{2}$. The temporal evolutions of the scale factor and of the energy density are shown in Figs. 11 and 12

\section{B. Stiff matter and anti-dark energy}

We consider a universe made of stiff matter and antidark energy. In the absence of matter $\left(\Omega_{m, 0}=0\right)$, we get

$$
\begin{gathered}
\frac{a}{a_{0}}=\left(\frac{\Omega_{s, 0}}{\left|\Omega_{\Lambda, 0}\right|}\right)^{1 / 6} \sin ^{1 / 3}\left(3 \sqrt{\left|\Omega_{\Lambda, 0}\right|} H_{0} t\right), \\
\frac{\epsilon}{\epsilon_{0}}=\frac{\left|\Omega_{\Lambda, 0}\right|}{\tan ^{2}\left(3 \sqrt{\left|\Omega_{\Lambda, 0}\right|} H_{0} t\right)} .
\end{gathered}
$$

At $t=0$ the universe starts from a singularity at which the scale factor $a=0$ and the energy density $\epsilon=+\infty$. 
Between $t=0$ and $t=t_{1}$ where

$$
t_{1}=\frac{\pi}{6 \sqrt{\left|\Omega_{\Lambda, 0}\right|} H_{0}},
$$

the energy density decreases from $\epsilon=+\infty$ to $\epsilon=0$ and the scale factor increases from $a=0$ to $a=a_{f}$ where

$$
\frac{a_{f}}{a_{0}}=\left(\frac{\Omega_{s, 0}}{\left|\Omega_{\Lambda, 0}\right|}\right)^{1 / 6} .
$$

Between $t=t_{1}$ and $t=t_{2}=2 t_{1}$ the energy density increases from $\epsilon=0$ to $\epsilon=+\infty$ and the scale factor decreases from $a=a_{f}$ to $a=0$. This process continues periodically with a period $t_{2}$.

\section{Dust matter and anti-dark energy}

We consider a universe made of dust matter and antidark energy. In the absence of stiff matter $\left(\Omega_{s, 0}=0\right)$, we obtain

$$
\begin{gathered}
\frac{a}{a_{0}}=\left(\frac{\Omega_{m, 0}}{\left|\Omega_{\Lambda, 0}\right|}\right)^{1 / 3} \sin ^{2 / 3}\left(\frac{3}{2} \sqrt{\left|\Omega_{\Lambda, 0}\right|} H_{0} t\right) \\
\frac{\epsilon}{\epsilon_{0}}=\frac{\left|\Omega_{\Lambda, 0}\right|}{\tan ^{2}\left(\frac{3}{2} \sqrt{\left|\Omega_{\Lambda, 0}\right|} H_{0} t\right)} .
\end{gathered}
$$

At $t=0$ the universe starts from a singularity at which the scale factor $a=0$ and the energy density $\epsilon=+\infty$. Between $t=0$ and $t=t_{1}$ where

$$
t_{1}=\frac{\pi}{3 \sqrt{\left|\Omega_{\Lambda, 0}\right|} H_{0}},
$$

the energy density decreases from $\epsilon=+\infty$ to $\epsilon=0$ and the scale factor increases from $a=0$ to $a=a_{f}$ where

$$
\frac{a_{f}}{a_{0}}=\left(\frac{\Omega_{m, 0}}{\left|\Omega_{\Lambda, 0}\right|}\right)^{1 / 3}
$$

Between $t=t_{1}$ and $t=t_{2}=2 t_{1}$ the energy density increases from $\epsilon=0$ to $\epsilon=+\infty$ and the scale factor decreases from $a=a_{f}$ to $a=0$. This process continues periodically with a period $t_{2}$. This solution corresponds to the anti- $\Lambda$ CDM model.

\section{THE CASE $\Omega_{s, 0} \leq 0$ AND $\Omega_{\Lambda, 0} \leq 0$}

We consider the case of a negative stiff energy density $\left(\Omega_{s, 0} \leq 0\right)$ and a negative cosmological constant $\left(\Omega_{\Lambda, 0} \leq\right.$ $0)$. The total energy density is

$$
\frac{\epsilon}{\epsilon_{0}}=-\frac{\left|\Omega_{s, 0}\right|}{\left(a / a_{0}\right)^{6}}+\frac{\Omega_{m, 0}}{\left(a / a_{0}\right)^{3}}-\left|\Omega_{\Lambda, 0}\right| .
$$

If $\left|\Omega_{s, 0}\right|>\Omega_{m, 0}^{2} /\left(4\left|\Omega_{\Lambda, 0}\right|\right)$ the energy density is always negative so this situation is not possible. Therefore, we assume $\left|\Omega_{s, 0}\right| \leq \Omega_{m, 0}^{2} /\left(4\left|\Omega_{\Lambda, 0}\right|\right)$. In that case, the energy density is positive for $a_{i} \leq a \leq a_{f}$ with

$$
\frac{a_{i}}{a_{0}}=\left(\frac{\Omega_{m, 0}-\sqrt{\Delta}}{2\left|\Omega_{\Lambda, 0}\right|}\right)^{1 / 3}
$$

and

$$
\frac{a_{f}}{a_{0}}=\left(\frac{\Omega_{m, 0}+\sqrt{\Delta}}{2\left|\Omega_{\Lambda, 0}\right|}\right)^{1 / 3},
$$

where we have defined

$$
\Delta=\Omega_{m, 0}^{2}-4\left|\Omega_{\Lambda, 0}\right|\left|\Omega_{s, 0}\right|
$$

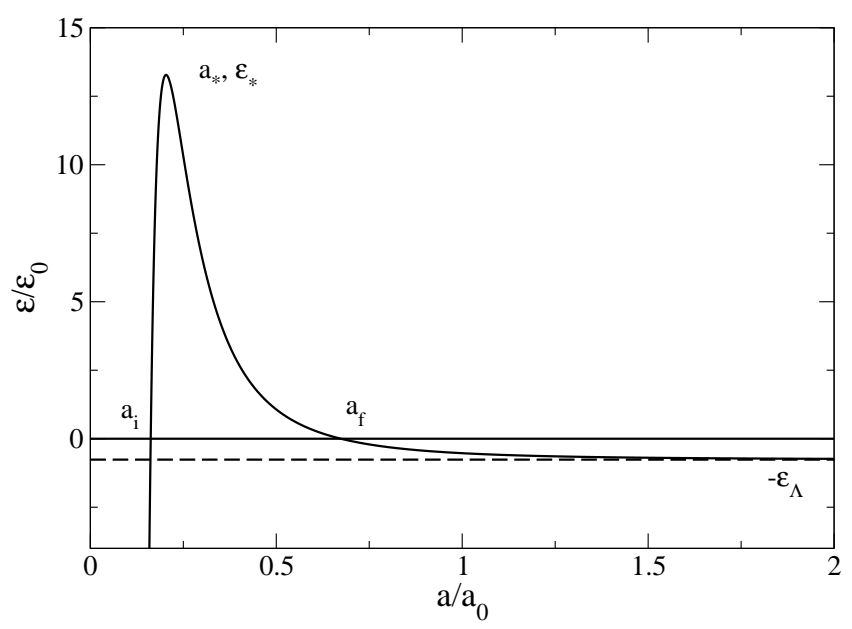

FIG. 13: Energy density as a function of the scale factor. We have taken $\Omega_{m, 0}=0.237, \Omega_{\Lambda, 0}=-0.763$, and $\Omega_{s, 0}=-10^{-3}$.

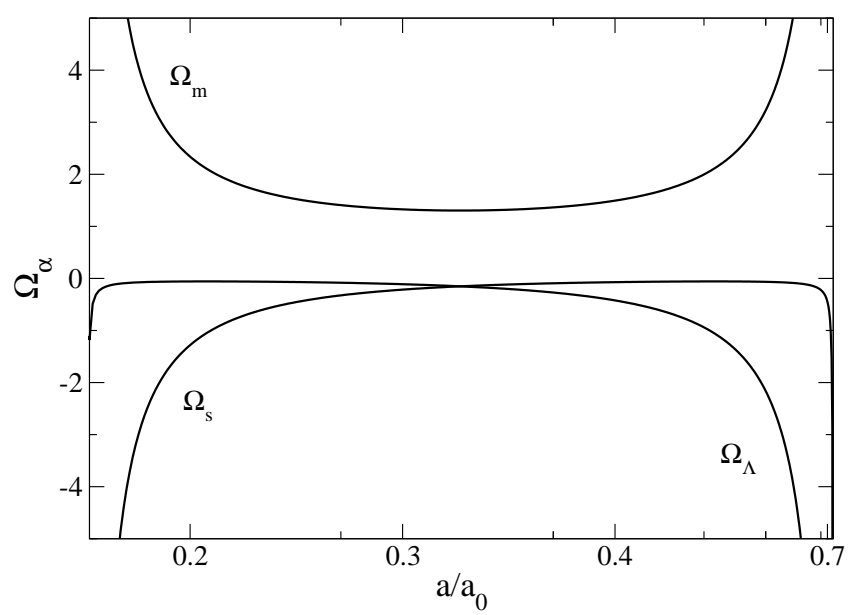

FIG. 14: Evolution of the proportion $\Omega_{\alpha}=\epsilon_{\alpha} / \epsilon$ of the different components of the universe with the scale factor. 
The energy density starts from $\epsilon=0$ at $a=a_{i}$, increases, reaches a maximum at

$$
\frac{a_{*}}{a_{0}}=\left(\frac{2\left|\Omega_{s, 0}\right|}{\Omega_{m, 0}}\right)^{1 / 3}, \quad \frac{\epsilon_{*}}{\epsilon_{0}}=\frac{\Delta}{4\left|\Omega_{s, 0}\right|},
$$

decreases, and reaches $\epsilon=0$ at $a=a_{f}$. The relation between the energy density and the scale factor is shown in Fig. 13. The proportions of stiff matter, dust matter and dark energy as a function of the scale factor are shown in Fig. 14.

\section{A. Anti-stiff matter, dust matter, and anti-dark energy}

We consider a universe made of anti-stiff matter, dust matter, and anti-dark energy. From Eqs. (55) and (56) with $\Omega_{\Lambda, 0}<0$, we get

$$
\frac{a}{a_{0}}=\left[\frac{\Omega_{m, 0}}{2\left|\Omega_{\Lambda, 0}\right|}-\frac{\sqrt{\Delta}}{2\left|\Omega_{\Lambda, 0}\right|} \cos \left(3 \sqrt{\left|\Omega_{\Lambda, 0}\right|} H_{0} t\right)\right]^{1 / 3},
$$

and

$$
\left(\frac{a}{a_{0}}\right)^{3} \frac{H}{H_{0}}=\frac{\sqrt{\Delta}}{2 \sqrt{\left|\Omega_{\Lambda, 0}\right|}} \sin \left(3 \sqrt{\left|\Omega_{\Lambda, 0}\right|} H_{0} t\right) .
$$

The energy density is given by Eq. (30) where $H / H_{0}$ can be obtained from Eq. (87) with Eq. (86).

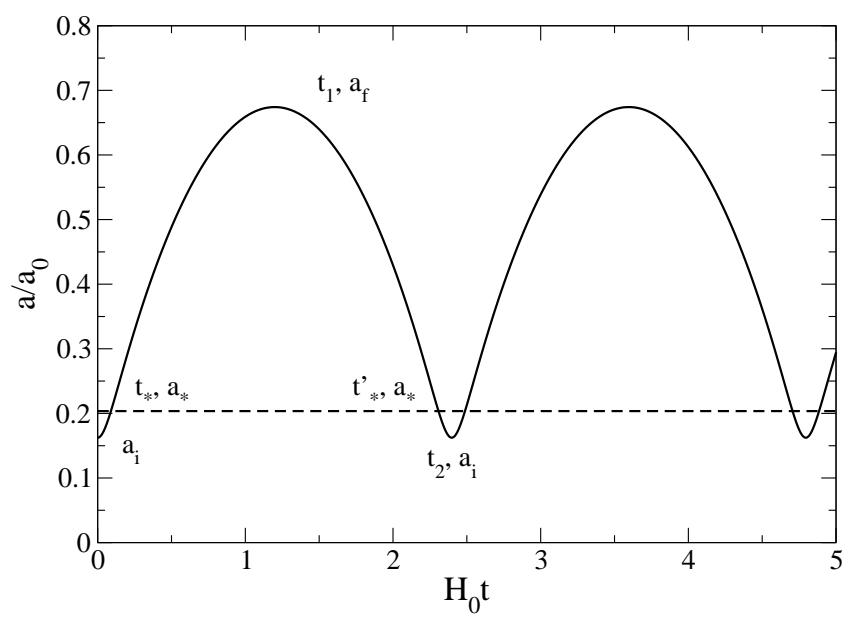

FIG. 15: Evolution of the scale factor as a function of time.

At $t=0$ the universe starts from a non-singular state at which the scale factor $a=a_{i}$ and the energy density $\epsilon=0$. Between $t=0$ and $t=t_{*}$ where

$$
t_{*}=\frac{\cos ^{-1}\left(\sqrt{\Delta} / \Omega_{m, 0}\right)}{3 \sqrt{\left|\Omega_{\Lambda, 0}\right|} H_{0}}
$$

the energy density increases from $\epsilon=0$ to its maximum value $\epsilon=\epsilon_{*}$ and the scale factor increases from $a=a_{i}$ to

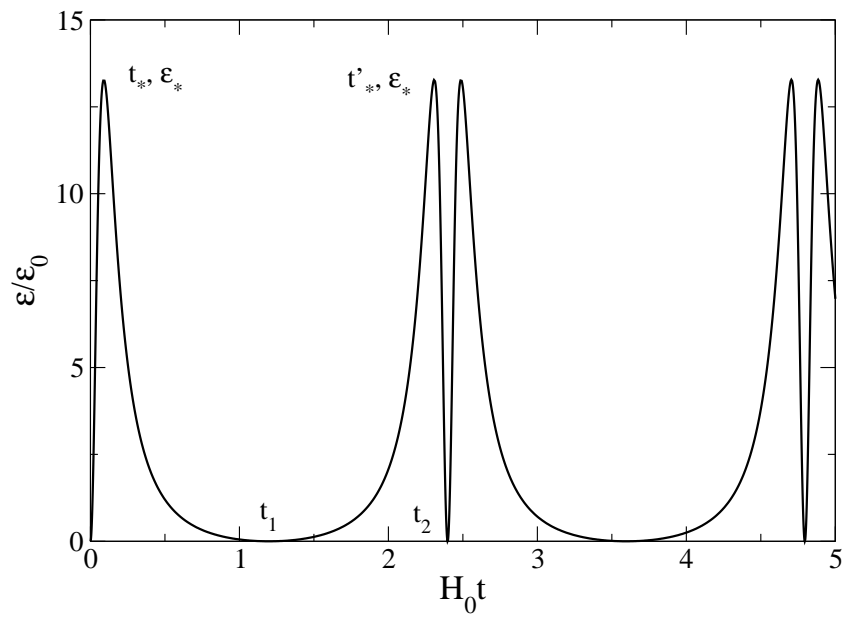

FIG. 16: Evolution of the energy density as a function of time.

$a=a_{*}$. Between $t=t_{*}$ and $t=t_{1}$ where

$$
t_{1}=\frac{\pi}{3 \sqrt{\left|\Omega_{\Lambda, 0}\right|} H_{0}}
$$

the energy density decreases from $\epsilon=\epsilon_{*}$ to $\epsilon=0$ and the scale factor increases from $a=a_{*}$ to $a=a_{f}$. Between $t=t_{1}$ and $t_{*}^{\prime}$ where

$$
t_{*}^{\prime}=\frac{2 \pi-\cos ^{-1}\left(\sqrt{\Delta} / \Omega_{m, 0}\right)}{3 \sqrt{\left|\Omega_{\Lambda, 0}\right|} H_{0}},
$$

the energy density increases from $\epsilon=0$ to $\epsilon=\epsilon_{*}$ and the scale factor decreases from $a=a_{f}$ to $a=a_{*}$. Between $t_{*}^{\prime}$ and $t_{2}=2 t_{1}$ the energy density decreases from $\epsilon=\epsilon_{*}$ to $\epsilon=0$ and the scale factor decreases from $a=a_{*}$ to $a=a_{i}$. This process continues periodically with a period $t_{2}$. The temporal evolutions of the scale factor and of the energy density are shown in Figs. 15 and 16.

\section{SOME ANALYTICAL SOLUTIONS INCLUDING THE RADIATION ERA}

We now come back to the general equation (31) including the contribution of radiation and provide some particular analytical solutions.

\section{A. Stiff matter and radiation}

We consider a universe made of stiff matter and radiation. The total energy is

$$
\frac{\epsilon}{\epsilon_{0}}=\frac{\Omega_{s, 0}}{\left(a / a_{0}\right)^{6}}+\frac{\Omega_{\text {rad }, 0}}{\left(a / a_{0}\right)^{4}} .
$$

The energy density starts from $\epsilon=+\infty$ at $a=a_{i}=0$ and decreases when $a$ increases. In the absence of dust 
matter and dark energy $\left(\Omega_{m, 0}=\Omega_{\Lambda, 0}=0\right)$ the integral in Eq. (31) can be performed analytically giving

$$
\begin{aligned}
& 2 \sqrt{\Omega_{\text {rad }, 0}} \frac{a}{a_{0}} \sqrt{\Omega_{s, 0}+\Omega_{r a d, 0}\left(\frac{a}{a_{0}}\right)^{2}}-2 \Omega_{s, 0} \\
& \times \ln \left[\Omega_{r a d, 0} \frac{a}{a_{0}}+\sqrt{\Omega_{\text {rad }, 0}} \sqrt{\Omega_{s, 0}+\Omega_{\text {rad }, 0}\left(\frac{a}{a_{0}}\right)^{2}}\right] \\
& +\Omega_{s, 0} \ln \left(\Omega_{s, 0} \Omega_{r a d, 0}\right)=4\left(\Omega_{r a d, 0}\right)^{3 / 2} H_{0} t .
\end{aligned}
$$

At $t=0$ the universe starts from a singular state at which the scale factor $a=0$ while the energy density $\epsilon \rightarrow+\infty$. The scale factor increases with time while the energy density decreases with time.

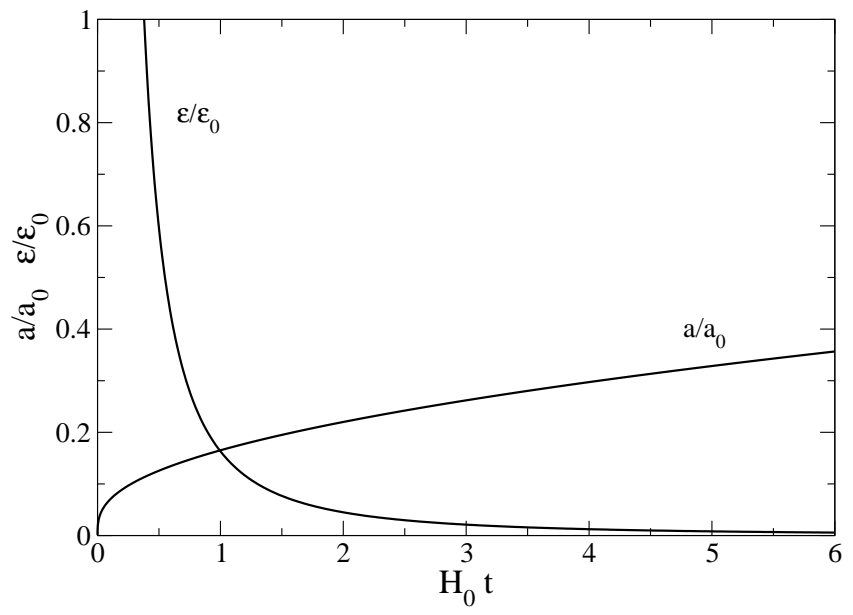

FIG. 17: Evolution of the scale factor and of the energy density as a function of time. We have taken $\Omega_{s, 0}=10^{-6}$ and $\Omega_{\text {rad }, 0}=8.4810^{-5}$ (here and in the following figure, we have chosen a relatively large value of the density of stiff matter $\Omega_{s, 0}$ for a better illustration of the results).

The temporal evolutions of the scale factor and of the energy density are shown in Fig. 17.

\section{B. Anti-stiff matter and radiation}

We consider a universe made of anti-stiff matter and radiation. The total energy is

$$
\frac{\epsilon}{\epsilon_{0}}=-\frac{\left|\Omega_{s, 0}\right|}{\left(a / a_{0}\right)^{6}}+\frac{\Omega_{\text {rad }, 0}}{\left(a / a_{0}\right)^{4}} .
$$

The energy density is positive for $a \geq a_{i}$ with

$$
\frac{a_{i}}{a_{0}}=\left(\frac{\left|\Omega_{s, 0}\right|}{\Omega_{r a d, 0}}\right)^{1 / 2} .
$$

The energy density starts from $\epsilon=0$ at $a=a_{i}$, increases, reaches a maximum at

$$
\frac{a_{*}}{a_{0}}=\left(\frac{3\left|\Omega_{s, 0}\right|}{2 \Omega_{r a d, 0}}\right)^{1 / 2}, \quad \frac{\epsilon_{*}}{\epsilon_{0}}=\frac{4 \Omega_{r a d, 0}^{3}}{27\left|\Omega_{s, 0}\right|^{2}},
$$

and decreases. In the absence of dust matter and dark energy $\left(\Omega_{m, 0}=\Omega_{\Lambda, 0}=0\right)$ the integral in Eq. (31) can be performed analytically giving

$$
\begin{array}{r}
2 \sqrt{\Omega_{\text {rad }, 0}} \frac{a}{a_{0}} \sqrt{-\left|\Omega_{s, 0}\right|+\Omega_{\text {rad }, 0}\left(\frac{a}{a_{0}}\right)^{2}}+2\left|\Omega_{s, 0}\right| \\
\times \ln \left[\Omega_{\text {rad }, 0} \frac{a}{a_{0}}+\sqrt{\Omega_{\text {rad }, 0}} \sqrt{-\left|\Omega_{s, 0}\right|+\Omega_{\text {rad }, 0}\left(\frac{a}{a_{0}}\right)^{2}}\right] \\
-\left|\Omega_{s, 0}\right| \ln \left(\left|\Omega_{s, 0}\right| \Omega_{\text {rad }, 0}\right)=4\left(\Omega_{\text {rad }, 0}\right)^{3 / 2} H_{0} t .
\end{array}
$$

At $t=0$ the universe starts from a non-singular state at which the scale factor $a=a_{i}$ and the energy density $\epsilon=0$. The scale factor increases with time. The energy density starts from $\epsilon=0$, increases, reaches its maximum value $\epsilon_{*}$ at $t=t_{*}$ where

$$
t_{*}=\frac{\left|\Omega_{s, 0}\right|}{4 \Omega_{\text {rad }, 0}^{3 / 2} H_{0}}[\sqrt{3}+2 \ln (\sqrt{3}+1)-\ln 2],
$$

and decreases.

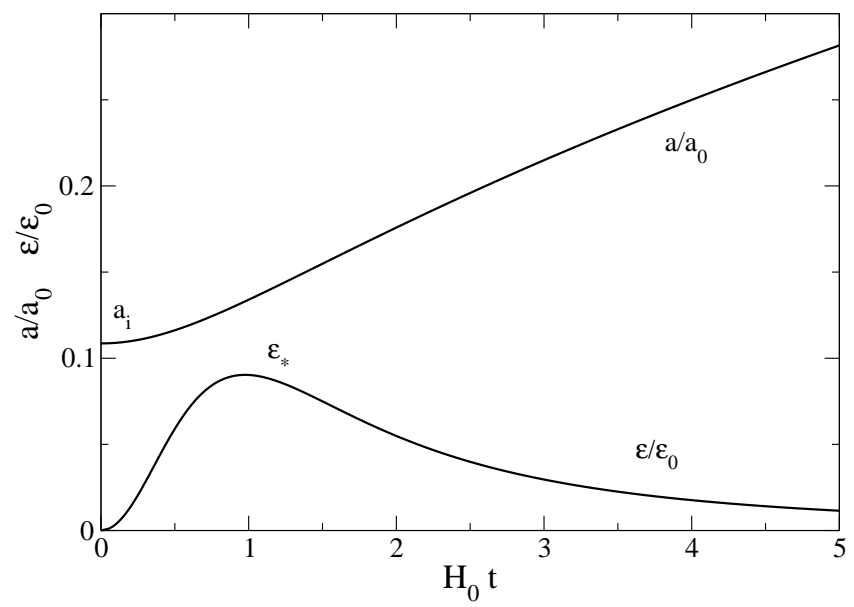

FIG. 18: Evolution of the scale factor and of the energy density as a function of time. We have taken $\Omega_{s, 0}=-10^{-6}$ and $\Omega_{\text {rad }, 0}=8.4810^{-5}$.

The temporal evolutions of the scale factor and of the energy density are shown in Fig. 18.

\section{Radiation}

We consider a universe made of radiation. In the absence of stiff matter, dust matter, and dark energy $\left(\Omega_{s, 0}=\Omega_{m, 0}=\Omega_{\Lambda, 0}=0\right)$ we get

$$
\frac{a}{a_{0}}=\Omega_{r a d, 0}^{1 / 4} \sqrt{2 H_{0} t}, \quad \frac{\epsilon}{\epsilon_{0}}=\frac{1}{\left(2 H_{0} t\right)^{2}} .
$$




\section{Radiation and dust matter}

We consider a universe made radiation and dust matter. The total energy is

$$
\frac{\epsilon}{\epsilon_{0}}=\frac{\Omega_{\text {rad,0}}}{\left(a / a_{0}\right)^{4}}+\frac{\Omega_{m, 0}}{\left(a / a_{0}\right)^{3}} .
$$

The energy density starts from $\epsilon=+\infty$ at $a=a_{i}=0$ and decreases as $a$ increases. In the absence of stiff matter and dark energy $\left(\Omega_{s, 0}=\Omega_{\Lambda, 0}=0\right)$ the integral in Eq. (31) can be performed analytically leading to

$$
\begin{aligned}
H_{0} t=-\frac{2}{3} \frac{1}{\left(\Omega_{m, 0}\right)^{1 / 2}}\left(\frac{2 \Omega_{\text {rad,0 }}}{\Omega_{m, 0}}-\right. & \left.\frac{a}{a_{0}}\right) \sqrt{\frac{\Omega_{r a d, 0}}{\Omega_{m, 0}}+\frac{a}{a_{0}}} \\
& +\frac{4}{3} \frac{\left(\Omega_{r a d, 0}\right)^{3 / 2}}{\left(\Omega_{m, 0}\right)^{2}} .
\end{aligned}
$$

Eq. (100) can also be written as

$$
\left(\frac{a}{a_{0}}\right)^{3}-3 \frac{\Omega_{\text {rad,0}}}{\Omega_{m, 0}}\left(\frac{a}{a_{0}}\right)^{2}=\frac{9}{4} \Omega_{m, 0} H_{0}^{2} t^{2}-6 \frac{\Omega_{r a d, 0}^{3 / 2}}{\Omega_{m, 0}} H_{0} t
$$

This is a cubic equation for $a / a_{0}$. At $t=0$ the universe starts from a singular state at which the scale factor $a=$ 0 while the energy density $\epsilon \rightarrow+\infty$. The scale factor increases with time while the energy density decreases with time.

\section{E. Radiation and dark energy}

We consider a universe made of radiation and dark energy. The total energy is

$$
\frac{\epsilon}{\epsilon_{0}}=\frac{\Omega_{r a d, 0}}{\left(a / a_{0}\right)^{4}}+\Omega_{\Lambda, 0} .
$$

The energy density starts from $\epsilon=+\infty$ at $a=a_{i}=0$ and tends to $\epsilon_{\Lambda}$ when $a \rightarrow+\infty$. In the absence of stiff matter and dust matter $\left(\Omega_{s, 0}=\Omega_{m, 0}=0\right)$, we get

$$
\begin{gathered}
\frac{a}{a_{0}}=\left(\frac{\Omega_{\text {rad,0 }}}{\Omega_{\Lambda, 0}}\right)^{1 / 4} \sinh ^{1 / 2}\left(2 \sqrt{\Omega_{\Lambda, 0}} H_{0} t\right), \\
\frac{\epsilon}{\epsilon_{0}}=\frac{\Omega_{\Lambda, 0}}{\tanh ^{2}\left(2 \sqrt{\Omega_{\Lambda, 0}} H_{0} t\right)} .
\end{gathered}
$$

At $t=0$ the universe starts from a singular state at which the scale factor $a=0$ while the energy density $\epsilon=+\infty$. The scale factor increases with time while the energy density decreases with time and tends to $\epsilon_{\Lambda}$ for $t \rightarrow+\infty$.

\section{F. Radiation and anti-dark energy}

We consider a universe made of radiation and anti-dark energy. The total energy is

$$
\frac{\epsilon}{\epsilon_{0}}=\frac{\Omega_{r a d, 0}}{\left(a / a_{0}\right)^{4}}-\left|\Omega_{\Lambda, 0}\right|
$$

The energy density is positive for $a \leq a_{f}$ with

$$
\frac{a_{f}}{a_{0}}=\left(\frac{\Omega_{r a d, 0}}{\left|\Omega_{\Lambda, 0}\right|}\right)^{1 / 4}
$$

The energy density starts from $\epsilon=+\infty$ at $a=a_{i}=0$, decreases, and reaches $\epsilon=0$ at $a=a_{f}$. In the absence of stiff matter and dust matter $\left(\Omega_{s, 0}=\Omega_{m, 0}=0\right)$, we get

$$
\frac{a}{a_{0}}=\left(\frac{\Omega_{r a d, 0}}{\left|\Omega_{\Lambda, 0}\right|}\right)^{1 / 4} \sin ^{1 / 2}\left(2 \sqrt{\left|\Omega_{\Lambda, 0}\right|} H_{0} t\right)
$$

$$
\frac{\epsilon}{\epsilon_{0}}=\frac{\left|\Omega_{\Lambda, 0}\right|}{\tan ^{2}\left(2 \sqrt{\left|\Omega_{\Lambda, 0}\right|} H_{0} t\right)} .
$$

At $t=0$ the universe starts from a singular state at which the scale factor $a=0$ while the energy density $\epsilon=+\infty$. Between $t=0$ and $t=t_{1}$ where

$$
t_{1}=\frac{\pi}{4 \sqrt{\left|\Omega_{\Lambda, 0}\right|} H_{0}},
$$

the energy density decreases from $\epsilon=+\infty$ to $\epsilon=0$ and the scale factor increases from $a=0$ to $a=a_{f}$. Between $t_{1}$ and $t_{2}=2 t_{1}$ the energy density increases from $\epsilon=0$ to $\epsilon=+\infty$ and the scale factor decreases from $a=a_{f}$ to $a=0$. This process continues periodically with a period $t_{2}$.

\section{CONCLUSION}

In this paper, we have obtained analytical solutions of the Friedmann equations for a universe undergoing a primordial stiff matter era. This stiff matter era appears in the cosmological model of Zel'dovich [1, 2] in which the universe is made of a cold gas of baryons. It also appears in certain models of relativistic BECs with a stiff equation of state [3]. In this paper, we have studied the evolution of the homogeneous background. For the sake of generality, we have considered a positive or a negative energy density of the stiff matter (leading to singular or non-singular models of universe) and a positive or a negative value of the cosmological constant (leading to expanding or oscillating models of universe). In a future work, we shall consider the evolution of the perturbations in these models. 


\section{Appendix A: A generalization of the analytical solutions}

We consider a universe containing three noninteracting fluids, each of them described by a linear equation of state $p_{i}=\alpha_{i} \epsilon_{i}$ with $\alpha_{1}=\alpha, \alpha_{2}=(\alpha-1) / 2$, and $\alpha_{3}=-1$ (dark energy). Since $\alpha_{1} \leq 1$, we have $\alpha_{2} \leq 0$. Therefore, the second fluid necessarily has a negative (or a vanishing) pressure. On the other hand, the two fluids are either both normal $\left(\alpha_{1}>-1\right.$ and $\left.\alpha_{2}>-1\right)$ or both phantom-like $\left(\alpha_{1}<-1\right.$ and $\left.\alpha_{2}<-1\right)$. Some triplets $\left(\alpha_{1}, \alpha_{2}, \alpha_{3}\right)$ of physical interest are $(1,0,-1)$, $(0,-1 / 2,-1),(1 / 3,-1 / 3,-1)$, and $(-1,-1,-1)$. The first case $\left(\alpha_{1}, \alpha_{2}, \alpha_{3}\right)=(1,0,-1)$ corresponds to a universe made of stiff matter, dust matter, and dark energy as studied in the main part of this paper. The total energy density is

$$
\frac{\epsilon}{\epsilon_{0}}=\frac{\Omega_{s, 0}}{\left(a / a_{0}\right)^{3(1+\alpha)}}+\frac{\Omega_{m, 0}}{\left(a / a_{0}\right)^{\frac{3}{2}(1+\alpha)}}+\Omega_{\Lambda, 0} .
$$

The Friedmann equation (19) takes the form

$$
\int_{a_{i} / a_{0}}^{a / a_{0}} \frac{d x}{x \sqrt{\frac{\Omega_{s, 0}}{x^{3(1+\alpha)}}+\frac{\Omega_{m, 0}}{x^{\frac{3}{2}(1+\alpha)}}+\Omega_{\Lambda, 0}}}=H_{0} t
$$

With the change of variables $X=x^{(1+\alpha) / 2}$, we obtain

$$
\int_{\left(a_{i} / a_{0}\right)^{(1+\alpha) / 2}}^{\left(a / a_{0}\right)^{(1+\alpha) / 2}} \frac{d X}{X \sqrt{\frac{\Omega_{s, 0}}{X^{6}}+\frac{\Omega_{m, 0}}{X^{3}}+\Omega_{\Lambda, 0}}}=\frac{1+\alpha}{2} H_{0} t
$$

where we recognize the integral in Eq. (32). As a result, the evolution of the scale factor and of the energy density of a universe containing three non-interacting fluids with linear coefficient $\alpha_{1}=\alpha, \alpha_{2}=(\alpha-1) / 2$, and $\alpha_{3}=-1$ are given by the equations of the main part of this paper with the substitutions $a / a_{0} \rightarrow\left(a / a_{0}\right)^{(1+\alpha) / 2}$ and $H_{0} t \rightarrow$ $(1+\alpha) H_{0} t / 2$.

\section{Appendix B: General polytropic equation of state}

In the main part of the paper (see also [3]), we have considered a polytropic equation of state of index $n=1$ (i.e. $\gamma=2$ ). This is the equation of state that appears in the model of Zel'dovich [1, 2]. This is also the standard equation of state of a self-interacting BEC at $T=0$ [26]. More generally, we can consider the polytropic equation of state [5]:

$$
P=K \rho^{\gamma}, \quad \gamma=1+\frac{1}{n},
$$

with arbitrary $\gamma$. For example, this equation of state can be derived from the Gross-Pitaevskii equation

$$
i \hbar \frac{\partial \psi}{\partial t}=-\frac{\hbar^{2}}{2 m} \Delta \psi+m\left(\Phi+\frac{K \gamma}{\gamma-1}(N m)^{\gamma-1}|\psi|^{2(\gamma-1)}\right) \psi,
$$

for a $\mathrm{BEC}$ at $T=0$ with an arbitrary nonlinearity [29].

We assume that the universe is filled with a relativistic fluid at $T=0$ described by the polytropic equation of state (B1). From Eq. (5), we find that the energy density is related to the rest-mass density by [3, 30]:

$$
\begin{gathered}
\epsilon=\rho c^{2}+K \rho \ln \left(\rho / \rho_{*}\right), \quad(\gamma=1), \\
\epsilon=\rho c^{2}+\frac{K}{\gamma-1} \rho^{\gamma}=\rho c^{2}+n P, \quad(\gamma \neq 1) .
\end{gathered}
$$

Combining Eq. (22) and Eqs. (B3) and (B4), we obtain for $\gamma=1$ :

$$
\epsilon=\rho_{0} c^{2}\left(\frac{a_{0}}{a}\right)^{3}+K \rho_{0}\left(\frac{a_{0}}{a}\right)^{3} \ln \left[\frac{\rho_{0}}{\rho_{*}}\left(\frac{a_{0}}{a}\right)^{3}\right]
$$

and for $\gamma \neq 1$ :

$$
\epsilon=\rho_{0} c^{2}\left(\frac{a_{0}}{a}\right)^{3}+\frac{K}{\gamma-1} \rho_{0}^{\gamma}\left(\frac{a_{0}}{a}\right)^{3 \gamma} .
$$

When $\gamma>1$ (i.e. $n>0$ ), we find that $P \sim \epsilon / n$ and $\epsilon \sim n K \rho^{\gamma} \propto a^{-3 \gamma}$ in the "early" universe ( $a$ small) and that $P \sim K\left(\epsilon / c^{2}\right)^{\gamma}$ and $\epsilon \sim \rho c^{2} \propto a^{-3}$ in the "late" universe $(a$ large). When $\gamma<1$ (i.e. $n<0$ ), we find that $P \sim K\left(\epsilon / c^{2}\right)^{\gamma}$ and $\epsilon \sim \rho c^{2} \propto a^{-3}$ in the "early" universe ( $a$ small) and that $P \sim \epsilon / n$ and $\epsilon \sim n K \rho^{\gamma} \propto a^{-3 \gamma}$ in the "late" universe ( $a$ large).

Introducing appropriate notations, the Friedmann equation (19) can be written as

$$
\frac{H}{H_{0}}=\sqrt{\frac{\Omega_{m, 0}^{\prime} \ln \left[\rho_{0}\left(a_{0} / a\right)^{3}\right]}{\ln \rho_{0}\left(a / a_{0}\right)^{3}}+\frac{\Omega_{m, 0}}{\left(a / a_{0}\right)^{3}}+\Omega_{\Lambda, 0}}
$$

for $\gamma=1$, and as

$$
\frac{H}{H_{0}}=\sqrt{\frac{\Omega_{\gamma, 0}}{\left(a / a_{0}\right)^{3 \gamma}}+\frac{\Omega_{m, 0}}{\left(a / a_{0}\right)^{3}}+\Omega_{\Lambda, 0}}
$$

for $\gamma \neq 1$.

For the polytropic equation of state (B1), our treatment shows that the energy density (B6) is the sum of two terms. An ordinary term $\epsilon_{m} \propto a^{-3}$ equivalent to dust matter and a new term $\epsilon_{\gamma} \propto a^{-3 \gamma}$ depending on the polytropic index $\gamma$. For $\gamma=2$ (i.e. $n=1$ ), the new term is equivalent to stiff matter $\left(\epsilon_{s} \propto a^{-6}\right)$ as discussed in the main part of the paper. For $\gamma=0$ (i.e. $n=-1$ ), the new term is equivalent to dark energy $\left(\epsilon_{\Lambda} \propto 1\right)$, as already noted in [31, 32] when the pressure is constant and negative. For $\gamma=4 / 3$ (i.e. $n=3$ ) the new term is equivalent to the radiation of an ultra-relativistic gas $\left(\epsilon_{\text {rad }} \propto a^{-4}\right)$.

More generally, according to Eq. (5), the new term that appears in the energy equation is equal to the internal energy

$$
\epsilon_{n e w}=u(\rho)=\rho \int_{0}^{\rho} \frac{P\left(\rho^{\prime}\right)}{\rho^{\prime 2}} d \rho^{\prime} .
$$


This relation clearly shows that the new term is related to pressure effects. When $P \simeq$ Cst, corresponding to $\gamma \rightarrow 0$ in the polytropic model, we suggest that this new term describes dark energy. Our procedure may be used to obtain generalized models of dark energy by considering different expressions of $P(\rho)$. In our approach, we have a single "dark fluid" described by an equation of state $P(\rho)$ "unifying" dark matter and dark energy. We suggest that this dark fluid may be in the form of relativistic self-interacting BECs at $T=0$ although other possibilities may be contemplated. If we assume that the energy density $\epsilon_{\text {new }}$ of the new term is positive (in agreement with the observations), we conclude from Eq. (B4) that the pressure must be positive for $\gamma>1$ and negative for $\gamma<1$. In particular, for the index $\gamma \rightarrow 0$ describing dark energy, the pressure must be negative. In the BEC interpretation, the pressure is negative when the self-interaction is attractive $(K<0)$. This may justify equations of state with negative pressure (such as the equation of state of dark energy) as already suggested in [29].

It may also be relevant to consider the logotropic equation of state 33 , 34]:

$$
P=A \ln \left(\rho / \rho_{*}\right),
$$

which can be viewed as the limiting form of a polytrope of index $\gamma \rightarrow 0(n \rightarrow-1)$ with $K \rightarrow \infty$ such that $A=K \gamma$ is finite [34]. In the case of BECs, using the general relations of Ref. [29], this equation of state can be derived from a Gross-Pitaevskii equation of the form

$$
i \hbar \frac{\partial \psi}{\partial t}=-\frac{\hbar^{2}}{2 m} \Delta \psi+m\left(\Phi-\frac{A}{N m|\psi|^{2}}\right) \psi,
$$

i.e. with the exponent -2 (inverted potential) instead of +2 in the usual Gross-Pitaevskii equation. This equation can also be obtained as the limiting form of Eq. (B2) when $\gamma \rightarrow 0$ and $K \rightarrow \infty$ with $A=K \gamma$ finite. For the logotropic equation of state (B10), the energy relation (5) becomes

$$
\epsilon=\rho c^{2}-A \ln \left(\frac{\rho}{\rho_{*}}\right)-A .
$$

Combining Eq. (22) and Eq. (B12), we obtain

$$
\epsilon=\rho_{0} c^{2}\left(\frac{a_{0}}{a}\right)^{3}-A \ln \left[\frac{\rho_{0}}{\rho_{*}}\left(\frac{a_{0}}{a}\right)^{3}\right]-A .
$$

For $a \rightarrow+\infty$, we get $\epsilon \sim 3 A \ln a$. Integrating the Friedmann equation (19) we obtain the "super de-Sitter" asymptotic behavior

$$
a \propto e^{\frac{2 \pi G A}{c^{2}} t^{2}}, \quad(t \rightarrow+\infty) .
$$

This model will be studied in more detail in a specific paper.
[1] Ya. B. Zel'dovich, Mon. Not. R. Astron. Soc. 160, 1 (1972)

[2] Ya. B. Zel'dovich, Soviet Phys. JETP 14, 1143 (1962)

[3] P.H. Chavanis, preprint

[4] P.H. Chavanis, Astron. Astrophys. 451, 109 (2006)

[5] S. Chandrasekhar, An Introduction to the Study of Stellar Structure (Dover, 1958)

[6] J.W. Lee, I. Koh, Phys. Rev. D 53, 2236 (1996)

[7] P.J.E. Peebles, Astrophys. J. 534, L127 (2000)

[8] J. Goodman, New Astronomy 5, 103 (2000)

[9] J. Lesgourgues, A. Arbey, P. Salati, New Astron. Rev. 46, 791 (2002)

[10] A. Arbey, J. Lesgourgues, P. Salati, Phys. Rev. D 68, 023511 (2003)

[11] C.G. Böhmer, T. Harko, J. Cosmol. Astropart. Phys. 06, 025 (2007)

[12] F. Briscese, Phys. Lett. B 696, 315 (2011)

[13] T. Harko, J. Cosmol. Astropart. Phys. 05, 022 (2011)

[14] M.O.C. Pires, J.C.C. de Souza, J. Cosmol. Astropart. Phys. 11 (2012) 024

[15] V.H. Robles, T. Matos, Monthly Not. Roy. Astron. Soc. 422, $282(2012)$

[16] T. Rindler-Daller, P. R. Shapiro, Monthly Not. Roy. Astron. Soc. 422, 135 (2012)

[17] V. Lora, J. Magaña, A. Bernal, F.J. Sánchez-Salcedo, E.K. Grebel, J. Cosmol. Astropart. Phys. 02, 011 (2012)

[18] A.X. González-Morales, A. Diez-Tejedor, L.A. Ureña-
López, O. Valenzuela, Phys. Rev. D 87, 021301(R) (2013)

[19] F.S. Guzmán, F.D. Lora-Clavijo, J.J. González-Avilés, F.J. Rivera-Paleo, J. Cosmol. Astropart. Phys. 09 (2013) 034

[20] T. Matos, A. Suárez, Europhys. Lett. 96, 56005 (2011)

[21] P.H. Chavanis, Phys. Rev. D 84, 043531 (2011)

[22] P.H. Chavanis, L. Delfini, Phys. Rev. D 84, 043532 (2011)

[23] E.P. Gross, Ann. of Phys. 4, 57 (1958); Nuovo Cimento 20, 454 (1961); J. Math. Phys. 4, 195 (1963)

[24] L.P. Pitaevskii, Sov. Phys. JETP 9, 830 (1959); ibid 13, 451 (1961)

[25] E. Madelung, Zeit. F. Phys. 40, 322 (1927)

[26] F. Dalfovo, S. Giorgini, L.P. Pitaevskii, S. Stringari, Rev. Mod. Phys. 71, 463 (1999)

[27] J. Binney, S. Tremaine, Galactic Dynamics (Princeton, NJ: Princeton University Press, 1987)

[28] S. Weinberg, Gravitation and Cosmology (John Wiley, 2002)

[29] P.H. Chavanis, Astron. Astrophys. 537, A127 (2012)

[30] R.F. Tooper, Astrophys. J. 142, 1541 (1965)

[31] P.H. Chavanis, Eur. Phys. J. Plus 129, 38 (2014)

[32] P.H. Chavanis, Eur. Phys. J. Plus 129, 222 (2014)

[33] D. McLaughlin, R. Pudritz, Astrophys. J. 476, 750 (1997)

[34] P.H. Chavanis, C. Sire, Physica A 375, 140 (2007) 